\title{
A SARS-CoV-2 neutralizing antibody with exceptional spike binding coverage and optimized therapeutic potentials
}

Yu Guo ( $\sim$ guoyu@nankai.edu.cn )

Nankai University https://orcid.org/0000-0002-8109-7515

Lisu Huang

Xinhua Hospital

Guangshun Zhang

Nankai University

Yanfeng Yao

Chinese Academy of Sciences

He Zhou

HiFibio(Hong Kong) Limited

\section{Shu Shen}

State Key Laboratory of Virology, Wuhan Institute of Virology, Chinese Academy of Sciences, Wuhan 430071 https://orcid.org/0000-0002-0013-5365

\section{Bingqing Shen}

HiFiBio(Hong Kong) Limited

\section{Bo Li}

Nankai University

Xin Li

Nankai University

Mingjie Chen

HiFiBio(Hong Kong) Limited

\section{Da Chen}

Nankai University

Jia Wu

HiFiBio(Hong Kong) Limited

\section{Dan Fu}

Nankai University

\section{Xinxin Zeng}

Xinhua Hospital

\section{Mingfang Feng}

HiFiBio(Hong Kong) Limited 


\section{Chunjiang Pi}

HiFiBio(Hong Kong) Limited

\section{Yuan Wang}

Nankai University

\section{Xingdong Zhou}

Nankai University https://orcid.org/0000-0001-8648-8948

\section{Minmin Lu}

HiFiBio(Hong Kong) Limited

\section{Yaohui Fang}

Wuhan Institute of Virology

\section{Yun-Yueh Lu}

HiFiBio(Hong Kong) Limited

\section{Xue Hu}

Wuhan Institute of Virology, Center for Biosafety Mega-Science, Chinese Academy of Sciences

\section{Shanshan Wang}

HiFiBio(Hong Kong) Limited

\section{Wanju Zhang}

Shanghai Public Health Clinical Center, Fudan University

\section{Qian Zhang}

HiFiBio(Hong Kong) Limited

\section{Ge Gao}

Wuhan Institute of Virology, Center for Biosafety Mega-Science, Chinese Academy of Sciences

Francisco Adrian

HiFiBio(Hong Kong) Limited

\section{Qisheng Wang}

Shanghai Synchrotron Radiation Facility, Shanghai Advanced Research Institute, Chinese Academy of

Sciences

\section{Feng Yu}

Shanghai Synchrotron Radiation Facility, Shanghai Advanced Research Institute, Chinese Academy of Sciences

\section{Yun Peng}

Wuhan Institute of Virology, Center for Biosafety Mega-Science, Chinese Academy of Sciences

\section{Alexander Gabibov}

Shemyakin-Ovchinnikov Institute of Bioorganic Chemistry

\section{Juan Min}

Wuhan Institute of Virology, Center for Biosafety Mega-Science, Chinese Academy of Sciences

\section{Yuhui Wang}

Nankai University

\section{Heyu Huang}


Xinhua Hospital

\section{Alexey Stepanov}

Shemyakin-Ovchinnikov Institute of Bioorganic Chemistry https://orcid.org/0000-0003-1616-4408

\section{Wei Zhang}

Nankai University https://orcid.org/0000-0001-8693-6747

\section{Yan Cai}

Tianjin International Joint Academy of Biotechnology \& Medicine

\section{Junwei Liu}

Tianjin International Joint Academy of Biotechnology \& Medicine

\section{Zhi-Ming Yuan}

Wuhan Institute of Virology https://orcid.org/0000-0002-3234-9616

\section{Chen Zhang}

Nankai University

\section{Zhiyong Lou}

Tsinghua University https://orcid.org/0000-0003-2728-881X

\section{Fei Deng}

State Key Laboratory of Virology, Wuhan Institute of Virology, Chinese Academy of Sciences, Wuhan 430071 https://orcid.org/0000-0002-5385-083X

\section{Hongkai Zhang}

Nankai University

\section{Chao Shan}

Chinese Academy of Sciences https://orcid.org/0000-0002-3953-3782

\section{Liang Schweizer}

HiFiBio(Hong Kong) Limited

\section{Kun Sun}

Xinhua Hospital

\section{Zihe Rao}

Tsinghua University https://orcid.org/0000-0001-9866-2384

\section{Article}

Keywords: SARS-CoV-2, spike glycoprotein, neutralizing antibody, antibody engineering, structure analysis, in vivo efficacy

Posted Date: November 14th, 2020

DOI: https://doi.org/10.21203/rs.3.rs-78945/v1

License: (c) (7) This work is licensed under a Creative Commons Attribution 4.0 International License. Read Full License 
Version of Record: A version of this preprint was published at Nature Communications on May 11th, 2021. See the published version at https://doi.org/10.1038/s41467-021-22926-2. 


\section{A SARS-CoV-2 neutralizing antibody with exceptional spike} binding coverage and optimized therapeutic potentials

Yu Guo ${ }^{1,7,12, *}, \$$, Lisu Huang ${ }^{2, \$}$, Guangshun Zhang ${ }^{1,4, \$}$, Yanfeng Yao ${ }^{5,} \$$, He Zhou ${ }^{3, \$}$, Shu Shen ${ }^{7}$, Bingqing Shen ${ }^{3}$, Bo Li ${ }^{11,4}$, Xin Li ${ }^{1,4}$, Mingjie Chen ${ }^{3}$, Da Chen ${ }^{1,4}$, Jia Wu ${ }^{3}$, Dan $\mathrm{Fu}^{1}$, Xinxin Zeng ${ }^{2}$, Mingfang Feng ${ }^{3}$, Chunjiang $\mathrm{Pi}^{3}$, Yuan Wang ${ }^{1,4}$, Xingdong Zhou ${ }^{1,4}$, Minmin Lu' ${ }^{3}$, Yaohui Fang ${ }^{7}$, Yun-Yueh Lu ${ }^{3}$, Xue Hu${ }^{7}$, Shanshan Wang ${ }^{3}$, Wanju Zhang ${ }^{2}$, Qian Zhang ${ }^{3}, \mathrm{Ge} \mathrm{Gao}^{5}$, Francisco Adrian $^{3}$, Qisheng Wang ${ }^{10}$, Feng $\mathrm{Yu}^{10}$, Yun Peng ${ }^{5}$, Alexander G. Gabibov ${ }^{11}$, Juan Min ${ }^{5}$, Yuhui Wang ${ }^{1,4}$, Heyu Huang ${ }^{2}$, Alexey Stepanov ${ }^{11}$, Wei Zhang ${ }^{1,4}$, Yan Cai ${ }^{6}$, Junwei Liu' ${ }^{6}$, Zhiming Yuan ${ }^{5}$, Chen Zhang ${ }^{1}$, Zhiyong Lou ${ }^{8,}{ }^{*}$, Fei Deng ${ }^{7,}{ }^{*}$, Hongkai Zhang ${ }^{1,4,9,} 12^{*}$, Chao Shan ${ }^{7,}{ }^{*}$, Liang Schweizer ${ }^{3,{ }^{*}}$, Kun Sun ${ }^{2,}{ }^{*}$, Zihe Rao ${ }^{1,4,}{ }^{*}$

1 State Key Laboratory of Medicinal Chemical Biology and College of Pharmacy, Nankai University, 38 Tongyan Road, Tianjin, 300071, China

2 Xinhua Hospital, Shanghai Jiao Tong University School of Medicine, Shanghai, China.

${ }^{3}$ HiFiBio (Hong Kong) Limited, 2 Ice House Street, Central, Hong Kong

${ }^{4}$ College of Life Science, Nankai University, 94 Weijin Road, Tianjin, 300071, China

${ }^{5}$ Center for Biosafety Mega-Science, Wuhan Institute of Virology, Chinese Academy of Sciences, Wuhan, Hubei 430071, China.

${ }^{6}$ Tianjin International Joint Academy of Biotechnology \& Medicine, Tianjin 300457, People's Republic of China

7 State Key Laboratory of Virology and National Virus Resource Center, Wuhan Institute of Virology, Chinese Academy of Sciences, Wuhan, Hubei 430071, China

8 MOE Key Laboratory of Protein Science \& Collaborative Innovation Center of Biotherapy, School of Medicine, Tsinghua University, Beijing, China

${ }^{9}$ Shanghai Institute for Advanced Immunochemical Studies, ShanghaiTech University, Shanghai, 201210, P.R. China

${ }^{10}$ Shanghai Synchrotron Radiation Facility, Shanghai Advanced Research Institute, Chinese Academy of Sciences, Shanghai 201204, China 
3311 Shemyakin-Ovchinnikov Institute of Bioorganic Chemistry, Russian Academy of 34 Sciences, 117997 Moscow, Russia

3512 Frontiers Science Center for Cell Responses, Nankai University, 94 Weijin Road, 36 Tianjin, 300071, China

37

38

$39 \$$ These authors contribute equally to this work

40 * Correspondence should be sent to: Z.R (raozh@mail.tsinghua.edu.cn),

41 K.S(sunkun@xinhuamed.com.cn), L.S (I.schweizer@hifibio.com);

42 C.S(shanchao@wh.iov.cn), H.Z(hongkai@nankai.edu.cn), ,F.D(df@wh.iov.cn),

43 Z.L(louzy@mail.tsinghua.edu.cn) and Y.G(guoyu@nankai.edu.cn) 


\section{Abstract}

45 The Coronavirus Disease of 2019 (COVID-19) pandemic caused by severe acute 46 respiratory syndrome coronavirus 2 (SARS-CoV-2) threatens global public health and

47 economy. Therapeutic options such as monoclonal antibodies (mAbs) against SARS-

48 CoV-2 are in urgent need. We have identified potent monoclonal antibodies binding to 49 SARS-CoV-2 Spike protein from COVID-19 convalescent patients and one of these 50 antibodies, P4A1, interacts directly and covers the majority of the Receptor Binding 51 Motif (RBM) of Spike receptor-binding domain (RBD), shown by high-resolution 52 complex structure analysis. We further demonstrated P4A1 binding and neutralizing 53 activities against wild type and mutant spike proteins. P4A1 was subsequently 54 engineered to reduce the potential risk for antibody-dependent enhancement (ADE) 55 of infection and to extend its half-life. The engineered mAb exhibits optimized 56 pharmacokinetic and safety profile, and results in complete viral clearance in a rhesus 57 monkey model of COVID-19 following a single injection.

\section{Keywords:}

59 SARS-CoV-2, spike glycoprotein, neutralizing antibody, antibody engineering, 60 structure analysis, in vivo efficacy 
61

62

63

64

\section{Introduction}

COVID-19 has recently emerged throughout the world as the largest pandemic of the 21st century, with over 35 million confirmed cases and over 1 million deaths worldwide as of Oct. $7^{\text {th }}, 2020$, which requires urgent prophylactic and treatment options. Like other Class I fusion proteins, the spike (S) glycoprotein forms homotrimers on the surface of SARS-CoV-2 virus particle, mediates the recognition and binding to the human receptor, angiotensin-converting enzyme 2 (hACE2), through its receptor-binding domain (RBD), and induces the virus-host cell membrane fusion, and serves as the primary target for interfering the virus entry process(1). A number of SARS-CoV-2 vaccines currently under development have shown successful induction of anti-viral antibodies in clinical trials (2-4). The final success of anti-SARS-CoV-2 vaccines may be depending on the quality of immune responses induced, particularly in elderly or individuals with pre-existing conditions, or the willingness to accept vaccination. Recent reports of SARS-CoV-2 reinfection in patients brought new challenges to the vaccine approaches.

To date, many efforts have been devoted to the discovery and development of SARS-CoV-2 neutralizing antibodies using various platforms, including antibody isolation and screening from $\operatorname{SARS}-\operatorname{CoV}(5,6)$ or SARS-CoV-2 convalescent patients(7-16), humanized mice $(17,18)$, and phage libraries $(19,20)$. Among them, several leading antibodies have entered the clinical trial stage. Of note, a major 
81 concern for the development of vaccine or neutralizing antibody therapies is the

82 potential risk of antibody-dependent enhancement (ADE) of infection $(21,22)$, which

83 also previously reported in infections of viruses such as dengue(23) and SARS-

$84 \operatorname{CoV}(24)$, and raised as a concern for SARS-CoV-2 countermeasures $(15,25)$. Another

85 aspect to consider is the ability of the evolving virus to mutate and escape from

86 treatments. To date, about 19 mutation sites in the Spike protein have been described

87 from an online database tracing $\sim 65 \mathrm{k}$ documented sequences

88 (https://cov.lanl.gov/content/index), of which D614G has become the most prevalent

89 form in the global pandemic(26), the development of potent treatments effective to

90 different SARS-CoV-2 mutants is urgently needed.

91 Results

To identify SARS-CoV-2 neutralizing antibodies from convalescent patients, we utilized a high-throughput single-cell B-cell receptor sequencing approach. SARS-

94 CoV-2 neutralizing antibodies were isolated from the two convalescent patients with

95 the highest titer against S protein (patient \#4 and patient \# 20) among 23 patients

96 studied (Fig. S1A). SARS-CoV-2 S protein binding B cells from PBMCs were enriched

97 with biotinylated S protein conjugated magnetic beads as probes as described (Fig.

98 S1C and S1D). Among the sequenced antibodies, P4A1, P20A2 and P20A3 were

99 shown to bind to the S1 subunit with sub-to-nM EC 50 s, but not to the S2 subunit, and

100 blocked the binding of the S1 subunit to hACE2 with $\mathrm{IC}_{50}$ values in the sub-to-nM range 
(Fig.1A). P4A1, P20A2, P20A3 neutralized the live SARS-CoV-2 infection of Vero-E6

cells with ND50 values of 5.212, 43.79, and $29.03 \mathrm{nM}$, respectively (Fig. 1B). According 103 to the ImmunoGenetics (IMGT) database-based analysis, IGHV3-53 was assigned as 104 the antibody P4A1's IGHV germline origin, which has been proved to be one of the 105 most frequent used germlines after SARS-CoV-2 infection in patients(16) (Fig. S3 and 106 S4A).

To gain further insight into the structural basis for the blocking and neutralizing mechanism of P4A1, the structure of the antibody Fab/Spike-RBD complex was resolved by X-ray crystallography at the resolution of $2.1 \AA$ (Fig.2A, Table.S1). Three 110 heavy chain complementarity-determining regions (HCDRs), two light chain 111 complementarity-determining regions (LCDRs), together with weak interaction from 112 the light chain framework region 3 (LFR3) constitute the interaction network with 113 SARS-CoV-2 RBD (Fig.2B). The buried surface area (BSA) from the heavy-chain 114 interaction in P4A1 (781 $\left.\AA^{2}\right)$ is relatively larger compared to reported neutralizing 115 antibodies including CC12.1 (723 $\left.\AA^{2}\right)$, CC12.3 $\left(698 \AA^{2}\right)(16)$, CB6(734 $\left.\AA^{2}\right)(14)$ and B38 $116\left(713 \AA^{2}\right)(14)$ (Fig.S3); However, the BSA from the light chain varies significantly, with $117 \mathrm{P} 4 \mathrm{~A} 1\left(577 \AA^{2}\right)$ being the largest compared to CC12.1 (566 $\left.\AA^{2}\right), \mathrm{CC} 12.3\left(176 \AA^{2}\right)(16)$, 118 CB6 $\left(334 \AA^{2}\right)(15)$ and B38 $\left(495 \AA^{2}\right)(14)$. Specifically, the interaction network is mainly 119 contributed by hydrogen bond and hydrophobic interaction. For the heavy chain, P4A1 120 HCDR1 and HCDR2 shares similar interaction pattern with SARS-CoV-2 Spike RBD 121 as CC12.1 and CC12.3, while these antibodies differ in their HCDR3 sequence, with 
122 Q100, E101 forming hydrogen bond with K417 and Y453, and the side chain of L102

123 inserts into the hydrophobic cavity formed by L485, F456 and Y489 (Fig. 2C, upper

124 panel); For the light chain, the hydrogen bond network is mediated by G28, S30, S31

125 W32 in LCDR1 with T500, N501, G502, Q498, Y449, and G496; and E90, N92, S93

126 in LCDR3 with Y505, and R403(Fig. 2C, lower panel). It is worth noting that the water

127 molecule plays more important role for light chain than it does for the heavy chain.

P4A1 Fab locates directly at the Receptor Binding Motif (RBM) region of SARS-

129 CoV-2 Spike RBD, hence inducing steric hindrance to the binding of receptor hACE2

130 (Fig. 2A). Except for residues G446 and Y449, 15 of the 17 residues identified as

131 SARS-CoV-2 Spike RBD-hACE2 binding sites(1) are within P4A1 epitope using the

132 distance of $<4 \AA$ as the cutoff, while Y449 is exactly $4 \AA$ from P4A1 (Fig. 2B, Table S2).

133 This represents one of the most extensive coverages of residues involved in the Spike-

134 hACE2 interaction to date and may explain potent neutralization activity for P4A1. This

135 broad coverage may suggest effectiveness against viral mutants as mutations to

136 hACE2 epitopes on Spike protein are rare in clinical isolates because of their

137 importance in viral infectivity. Structure analysis showed that all 8 residues with

138 reported mutations in the Spike RBD of clinical isolates (27), are mostly located far 139 away from P4A1 epitope (Fig. S4B), are expected to have no or minimal effects on the

140 binding and neutralization activity of P4A1. This was supported by the test of P4A1

141 binding ability to wild type, different RBD mutants and D614G variants of S1 protein 142 by SPR (Fig. 3A) or ELISA (Fig. S5), as well as neutralization of pseudoviruses 
143 expressing wildtype or D614G, V367F, D264Y or W436R variants of Spike protein with

144 similar IC50s (Fig. 3B).

To facilitate its clinical applications, P4A1 was further engineered as an IgG4

antibody (named as P4A1-2A) to reduce the risks of Fcy- or complement-receptor-

147 mediated ADE with modifications to reduce Fab-arm exchange(28), and to increase

148 antibody blood half-life in primates. Consistent with its isotype and Fc engineering,

149 P4A1-2A displayed 4-81 fold decrease in binding affinity to different FcyRs, no binding

150 to complement $\mathrm{C} 1 \mathrm{q}$, and enhanced affinity for FcRn when compared to its IgG1 form

151 P4A1 (Fig. 3C and S6). The effect of enhanced FcRn binding is further validated by

152 the longer serum half-life in cynomolgus monkey (Fig. S6). The prolonged half-life is

153 highly desired as it will be beneficial for long-term protection in individuals at high risk

154 of contracting SARS-CoV-2 infection during the pandemic. Furthermore, the safety of

155 P4A1-2A was demonstrated in a GLP Toxicity study where cynomolgus monkeys were

156 treated with 2 weekly i.v infusions of up to $300 \mathrm{mg} / \mathrm{kg} / \mathrm{dose}$ and no treatment-related

157 findings were observed in any of the tests performed, including cytokine levels, such

158 as IL-2, IL-6, IL-10, TNF- $\alpha$ and IFN- $\gamma$ at different timepoints. Besides, no tissue cross-

159 reactivity was observed for 37 different normal human tissues.

161 infection model in rhesus macaques (Figure 4A). Isotype control or P4A1-2A were

162 administered in a single intravenous (i.v.) infusion 1 day after intra-tracheal virus 163 inoculation at $1 \times 10^{5} \mathrm{TCID}_{50}$. Consistent with the previous report(29), in the isotype 
control (50 mg/kg) group, viral load using oropharyngeal swabs was at high level 1 day post-infection (d.p.i.), showing the colonization of virus, decreased 2 d.p.i., suggesting viral distribution, increased 3-4 d.p.i. and maintained at high level through 7 d.p.i indicating viral replication. Importantly, in 3 out of $3(50 \mathrm{mg} / \mathrm{kg})$ and 2 out the 3 $(10 \mathrm{mg} / \mathrm{kg})$ P4A1-2A-treated animals, the viral load dropped continuously to a level near or below the detection limit, and viral elimination occurred on 3-4 d.p.i., indicating effective blocking of viral propagation by P4A1-2A (Fig. 4B).

At necropsy, trachea, left and right bronchus and different lung lobes were

172 isolated, homogenized and tested for viral load. Substantial viral load was detected in

1735 or 6 out of the 9 tissues examined (including consistent viral presence in trachea,

174 left and right bronchus) with moderate to severe lung lesions similar to previous report

175 (29) in animals in the isotype control treatment group. In contrast, no viral load was

176 detected 6 or 7 d.p.i in any of these tissues examined and with to mild-moderate lung

177 lesions in the $50 \mathrm{mg} / \mathrm{kg}$ P4A1-2A-treated animals. In the $10 \mathrm{mg} / \mathrm{kg}$ P4A1-2A-treated 178 animals, virus was detected in only 1 of the 9 tissues examined 7 d.p.i and no viral 179 RNA detected in any of the lung tissues in all 3 animals (Fig. 4C, 4D and Fig S8). system than the currently reported antibodies(15) with lower i.v. dose of neutralizing 182 antibody used in similar NHP SARS-CoV-2 infection models. In addition to the potency 183 of P4A1-2A, a potentially better distribution to the respiratory tract associated with the 184 engineered IgG4 antibodies by previous work(30) may contribute to the total viral 
185 clearance in some of the animals. These data demonstrated that P4A1-2A was 186 effective despite reduced FcyR effector functions.

\section{Discussion}

High affinity neutralizing antibodies from convalescent patients may represent

some of the most efficient anti-viral weapons contributing to a patient recovery from

viral infection. Given that not every COVID-19 patient is able to produce high quality antibodies such as P4A1, this antibody along with antibody engineering to reduce potential safety concerns and to prolong the serum half-life, may provide durable protection to every patient and, especially, to individuals at high risk of viral infection.

194 The structural analysis demonstrates the molecular basis for neutralizing mechanism

195 of P4A1, whose epitope include most of the binding residues of hACE2 and exhibited 196 potent antiviral activities in vitro and in vivo. The broad coverage of hACE2 epitopes 197 may contribute to effectiveness to viral mutants, supported by activities against all 198 spike protein mutants tested, including the most prevalent variant D614G, with similar $199 \mathrm{EC}_{50} / \mathrm{IC}_{50}$ s compared to wild type protein.

With prolonged half-life, potential for a broad spectrum of viral neutralization,

201 P4A1-2A represents not only optimized therapeutic potency, but also an important 202 prophylactic option to safeguard the vulnerable or aged populations, complementary 203 to vaccine approaches. The comprehensive characterization of this antibody provides 
204 strong evidence for its promising potential to effectively treat and prevent COVID-19 205 in humans. 


\section{References}

207 1. J. Shang et al., Structural basis of receptor recognition by SARS-CoV-2. Nature

208

209

210

211

212

213

214

215

216

217

218

219

220

221

222

223

224

225

226

227

228

229

230

231

232

233

234

235

236

237

238

239

240

241

242

243

244

245 581, 221-224 (2020).

2. H. Wang et al., Development of an Inactivated Vaccine Candidate, BBIBP-CorV, with Potent Protection against SARS-CoV-2. Cell 182, 713-721 e719 (2020).

3. F. C. Zhu et al., Immunogenicity and safety of a recombinant adenovirus type5-vectored COVID-19 vaccine in healthy adults aged 18 years or older: a randomised, double-blind, placebo-controlled, phase 2 trial. Lancet 396, 479488 (2020).

4. P. M. Folegatti et al., Safety and immunogenicity of the ChAdOx1 nCoV-19 vaccine against SARS-CoV-2: a preliminary report of a phase $1 / 2$, single-blind, randomised controlled trial. Lancet 396, 467-478 (2020).

5. D. Pinto et al., Cross-neutralization of SARS-CoV-2 by a human monoclonal SARS-CoV antibody. Nature 583, 290-295 (2020).

6. A. Z. Wec et al., Broad neutralization of SARS-related viruses by human monoclonal antibodies. Science 369, 731-736 (2020).

7. D. Zhou et al., Structural basis for the neutralization of SARS-CoV-2 by an antibody from a convalescent patient. Nat Struct Mol Biol, (2020).

8. P. J. M. Brouwer et al., Potent neutralizing antibodies from COVID-19 patients define multiple targets of vulnerability. Science 369, 643-650 (2020).

9. Y. Cao et al., Potent Neutralizing Antibodies against SARS-CoV-2 Identified by High-Throughput Single-Cell Sequencing of Convalescent Patients' B Cells. Cell 182, 73-84 e16 (2020).

10. X. Chi et al., A neutralizing human antibody binds to the N-terminal domain of the Spike protein of SARS-CoV-2. Science 369, 650-655 (2020).

11. C. Kreer et al., Longitudinal Isolation of Potent Near-Germline SARS-CoV-2Neutralizing Antibodies from COVID-19 Patients. Cell 182, 843-854 e812 (2020).

12. D. F. Robbiani et al., Convergent antibody responses to SARS-CoV-2 in convalescent individuals. Nature 584, 437-442 (2020).

13. T. F. Rogers et al., Isolation of potent SARS-CoV-2 neutralizing antibodies and protection from disease in a small animal model. Science 369, 956-963 (2020).

14. Y. Wu et al., A noncompeting pair of human neutralizing antibodies block COVID-19 virus binding to its receptor ACE2. Science 368, 1274-1278 (2020).

15. R. Shi et al., A human neutralizing antibody targets the receptor-binding site of SARS-CoV-2. Nature 584, 120-124 (2020).

16. M. Yuan et al., Structural basis of a shared antibody response to SARS-CoV-2. Science 369, 1119-1123 (2020).

17. J. Hansen et al., Studies in humanized mice and convalescent humans yield a SARS-CoV-2 antibody cocktail. Science 369, 1010-1014 (2020). 
18. Z. Lv et al., Structural basis for neutralization of SARS-CoV-2 and SARS-CoV by a potent therapeutic antibody. Science, (2020).

19. H. A. Parray et al., Identification of an anti-SARS-CoV-2 receptor binding domain directed human monoclonal antibody from a naive semi-synthetic library. J Biol Chem, (2020).

20. Y. Wu et al., Identification of Human Single-Domain Antibodies against SARSCoV-2. Cell Host Microbe 27, 891-898 e895 (2020).

21. A. M. Arvin et al., A perspective on potential antibody-dependent enhancement of SARS-CoV-2. Nature 584, 353-363 (2020).

22. W. Hoepel et al., Anti-SARS-CoV-2 IgG from severely ill COVID-19 patients promotes macrophage hyper-inflammatory responses. bioRxiv, (2020).

23. L. C. Katzelnick et al., Antibody-dependent enhancement of severe dengue disease in humans. Science 358, 929-932 (2017).

24. M. S. Yip et al., Antibody-dependent infection of human macrophages by severe acute respiratory syndrome coronavirus. Virol J 11, 82 (2014).

25. L. M. Walker, D. R. Burton, Passive immunotherapy of viral infections: 'superantibodies' enter the fray. Nat Rev Immunol 18, 297-308 (2018).

26. B. Korber et al., Tracking Changes in SARS-CoV-2 Spike: Evidence that D614G Increases Infectivity of the COVID-19 Virus. Cell 182, 812-827 e819 (2020).

27. J. Ou et al., Emergence of RBD mutations in circulating SARS-CoV-2 strains enhancing the structural stability and human ACE2 receptor affinity of the spike protein. 2020.2003.2015.991844 (2020).

28. A. F. Labrijn et al., Therapeutic IgG4 antibodies engage in Fab-arm exchange with endogenous human IgG4 in vivo. Nat Biotechnol 27, 767-771 (2009).

29. C. Shan et al., Infection with novel coronavirus (SARS-CoV-2) causes pneumonia in Rhesus macaques. Cell Res 30, 670-677 (2020).

30. W. F. Dall'Acqua, P. A. Kiener, H. Wu, Properties of human IgG1s engineered for enhanced binding to the neonatal Fc receptor (FcRn). J Biol Chem 281, 23514-23524 (2006). 


\section{Correspondence}

279 Z.R (raozh@mail.tsinghua.edu.cn), K.S(sunkun@xinhuamed.com.cn), L.S

280 (I.schweizer@hifibio.com); C.S(shanchao@wh.iov.cn), H.Z(hongkai@nankai.edu.cn),

281 F.D(df@wh.iov.cn), Z.L(louzy@mail.tsinghua.edu.cn) and Y.G(guoyu@nankai.edu.cn)

\section{Acknowledgments}

283 We thank the running team of National Biosafety Laboratory, staffs from Center for 284 Instrumental Analysis and Metrology, Wuhan, Chinese Academy of Sciences, and staffs 285 from BL-17U1, Shanghai Synchrotron Radiation Facility. We thank Cheryl Cui and Jeff He 286 for their critical role in setting up collaboration among different institutions, Ning Zhang from 287 Red Avenue Foundation for her support.

288 This work was supported by the National Program on Key Research Project of China 289 (2018YFE0200400, 2018YFA0507203, 2017YFC840300, 2017YFA0504801 and 290 2020YFC0845801), the National Natural Science Foundation of China (NSFC) (31670731, 291 31870733), Projects of International Cooperation and Exchanges NSFC (grant 292 no.81520108019), Medical and Industrial Cross Research Foundation of Shanghai Jiao 293 Tong University (YG2020YQ27), and Science and Technology Innovation Achievements and 294 Team Building Foundation of Nankai University (grant no. ZB19500403, ZB19100123 and $29563201101)$ 


\section{Author contributions}

Z. R., K.S., L.S., H.-K.Z., Z.-Y. L., and Y.G. conceived the project. L.-S.H. X.-X.Z., W.-

298 J.Z., H.-Y.H. and K.S. collected the convalescent PBMC and serum and take responsibility 299 for the human ethic issue. Y.G., L.-S.H., G.-S.Z., B.-Q.S., M.-J.C., J.W., M.-F.F., B.L., D.F., 300 W.Z., Y.-H.W., Y.C., J.-W.L. and C.Z. performed gene construction, protein expression and 301 purification, and crystal screening and optimization. Y.G., X.L., Q.-S.W., Q.Z., F.Y., A.S., 302 and A.G.G. performed the collection of X-ray diffraction data and structure determination.

304 performed the in vitro binding assays and analysis data. S.S., Y.-H.F., and F.D. performed 305 live virus neutralization assays. C.S., Y.F.Y., X.H., G.G., Y.P., J.M., and Z.-M.Y. performed 306 animal assays. Y.G., H.-K.Z., L.S., H.Z., Z. R., K.S., and F.A. designed the experiments 307 and wrote the manuscript. All authors read and approved the contents of the manuscript.

\section{Competing interests}

309 L.S., B.-Q.S., H.Z., M.-J.C., Y.-Y.L.; L.S.H.; K.S. are the inventors of the pending patent 310 application filed on the reported antibodies. L.S., B.-Q.S., H.Z., M.-J.C., J.W., M.-F.F., C.311 J.P., M.-M.L., Y.-Y.L.; S.-S.W., Q.Z., F.A. employees of HiFiBio (Hong Kong) Ltd. Other 312 authors declare no competing interests. All reagents and information presented in this study 313 are available from corresponding authors upon reasonable request. 


\section{Figures and Figure Legends}

\section{Figure 1}

A
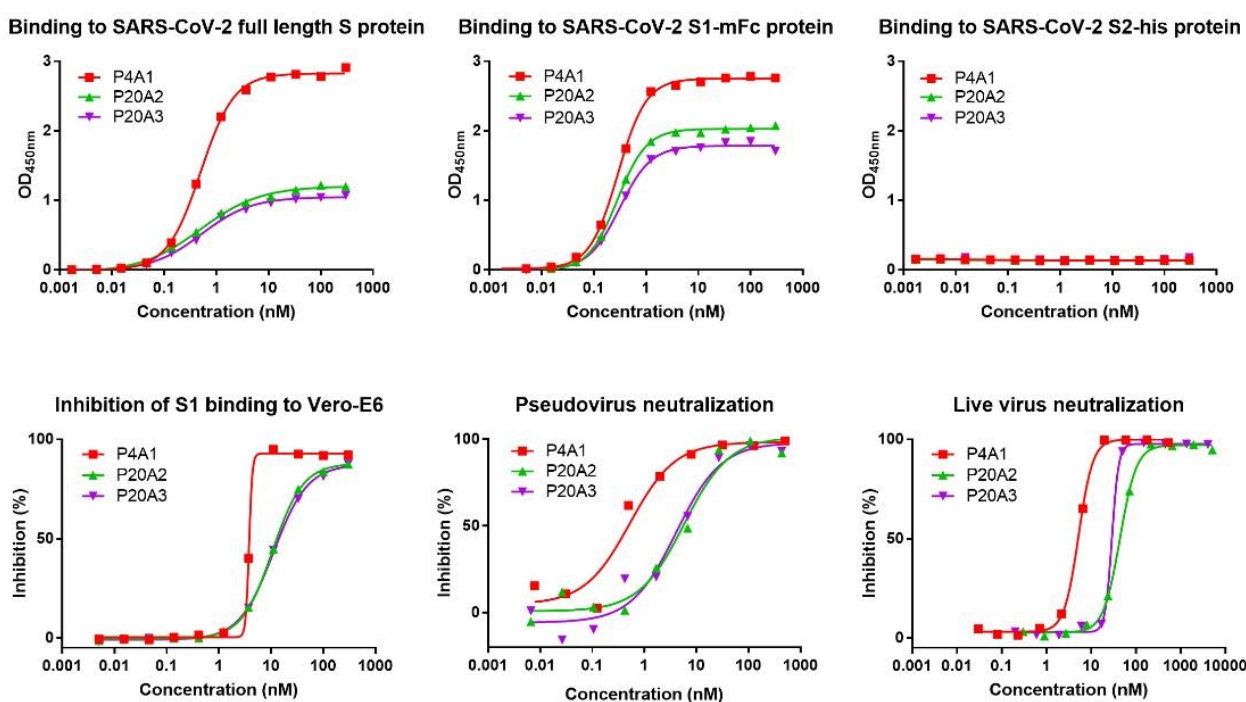

B

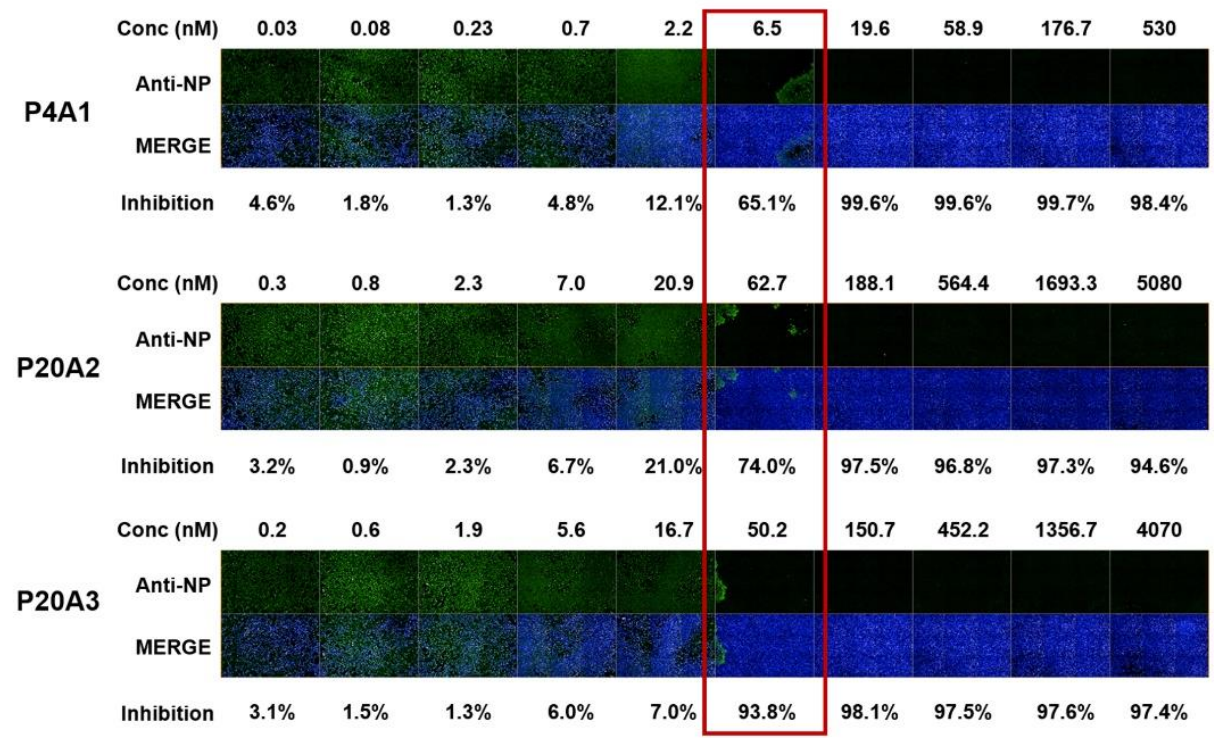

317 Figure 1. Characterization of neutralizing antibodies from convalescent patients. $(A)$

318 Characterization of SARS-CoV-2 S protein specific antibodies. (upper panels) Binding of

319 antibodies to the full-length S protein, S1 protein and S2 protein were evaluated by ELISA.

320 (lower left panel) Blockage of the binding of SARS-CoV-2 spike S1 protein to Vero E6 cell 
321 line by antibodies evaluated by flow cytometry. (Lower middle panel) Pseudovirus 322 neutralization assay in Huh-7 cell line. (lower right panel and B) SARS-CoV-2 live virus 323 neutralization assay. (B) Images of Vero E6 cells infected SARS-CoV-2 treated with 324 antibodies of different concentrations.

325 
Figure 2

A

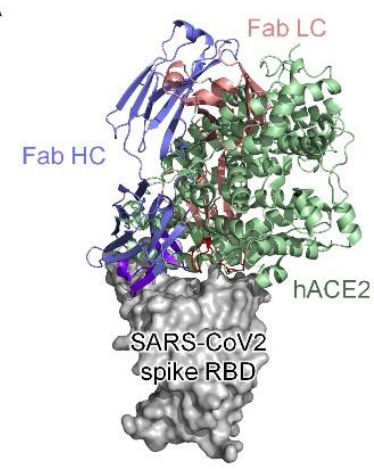

C
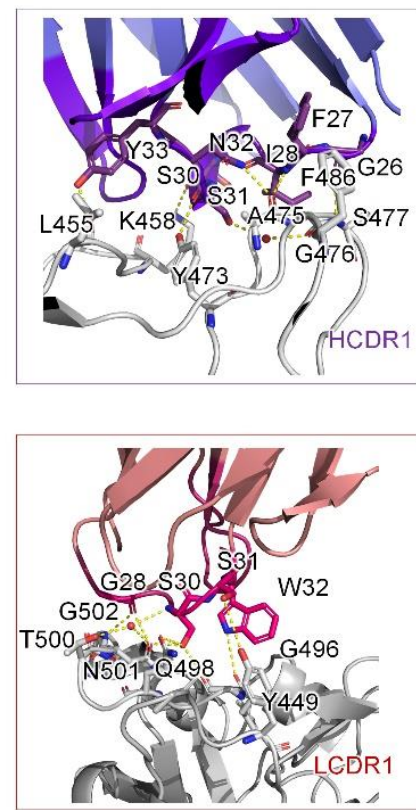

B

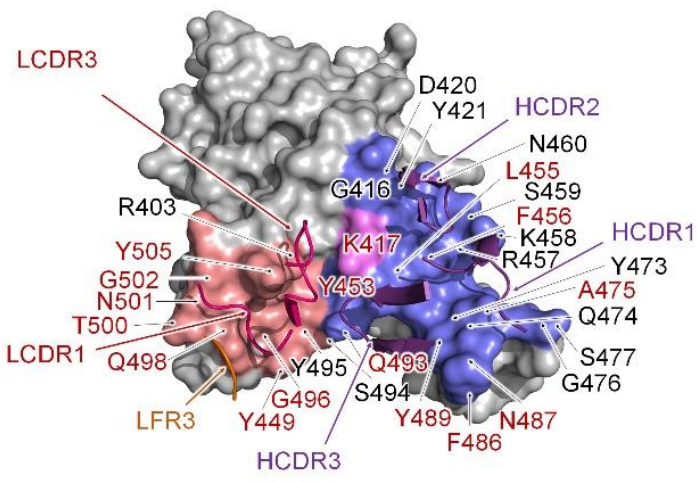

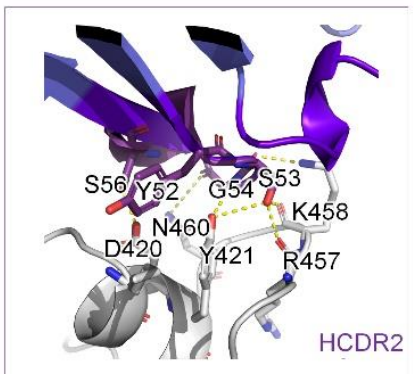
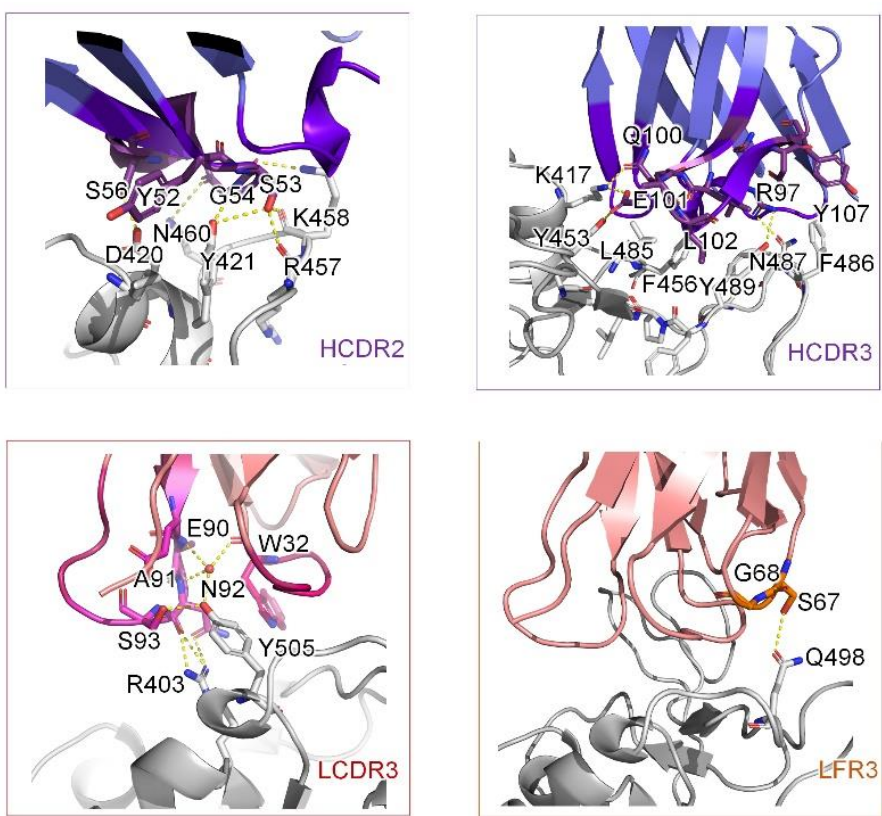

Figure 2. Structural analysis of P4A1 Fab and SARS-CoV-2 RBD complex. (A) The overall P4A1-Fab-RBD complex structure superimposed with the hACE2-RBD complex. The P4A1 heavy chain (colored slate blue), light chain (colored salmon red) and hACE (colored pale green) are displayed in cartoon representation. The SARS-CoV-2 RBD is colored in gray and displayed in surface representation. (B) The epitope of P4A1 shown in surface representation. The CDR loops of heavy chain (HCDR) and light chain (LCDR) are colored in purple and magenta, respectively. The epitopes from the heavy chain and light chain are colored in slate blue and salmon red, respectively. The only residue K417, which contacts with both heavy chain and light chain is colored in pink. The light chain frame region 3 (LFR3) is colored in orange. The identical residues on RBD shared in P4A1 and hACE2 binding are 
338 labelled in red. The residues are numbered according to SARS-CoV-2 RBD. (C) The detailed 339 interactions between SARS-CoV-2 RBD with HCDR, LCDR and LFR3. The residues are 340 shown in sticks with identical colors to $(B)$. 
A
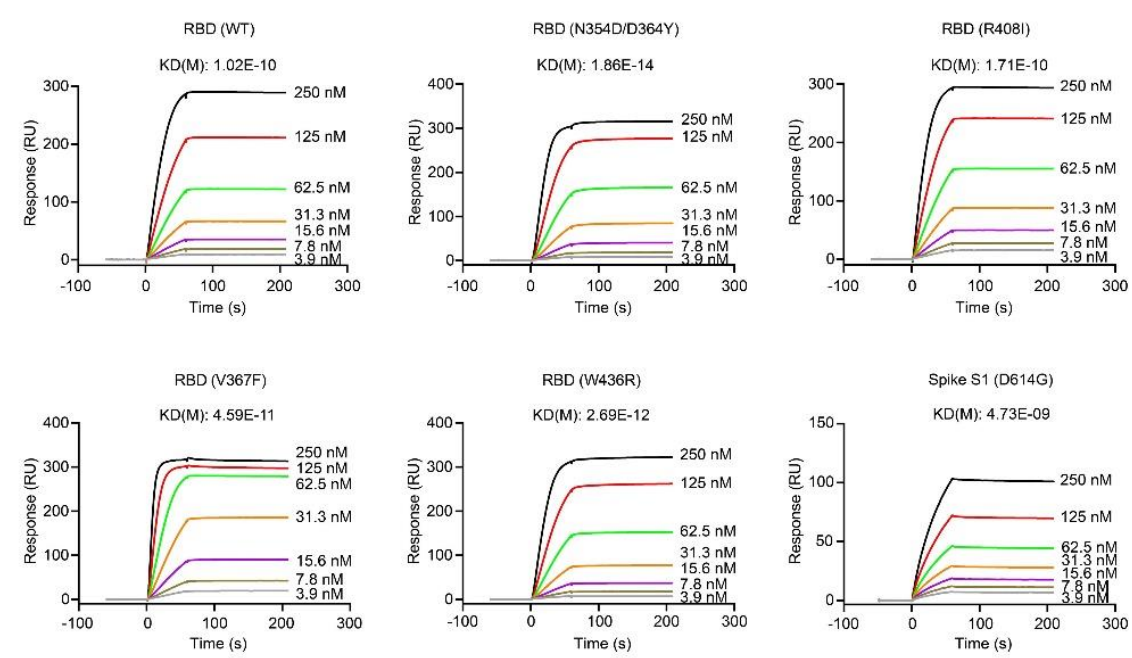

B

C

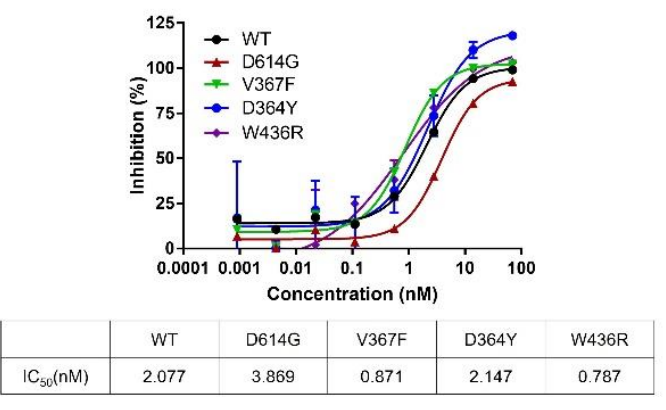

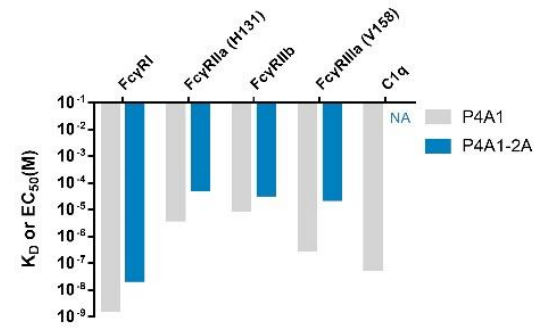

345 Figure 3. The activities of IgG4 antibody P4A1-2A to different SARS-CoV-2 S protein 346 mutants, FcyRs and C1q. (A) Binding of antibody P4A1 to SARS-CoV-2 S protein 347 N354D/D364Y, R408I, W436R, V367F or D614G mutants determined by SPR. (B) Pseudo 348 virus neutralization assay in hACE2 overexpressing HEK293 cells. (C) The binding affinity 349 of P4A1 and P4A1-2A for different human FcyRs and complement C1q. 


\section{Figure 4}

A

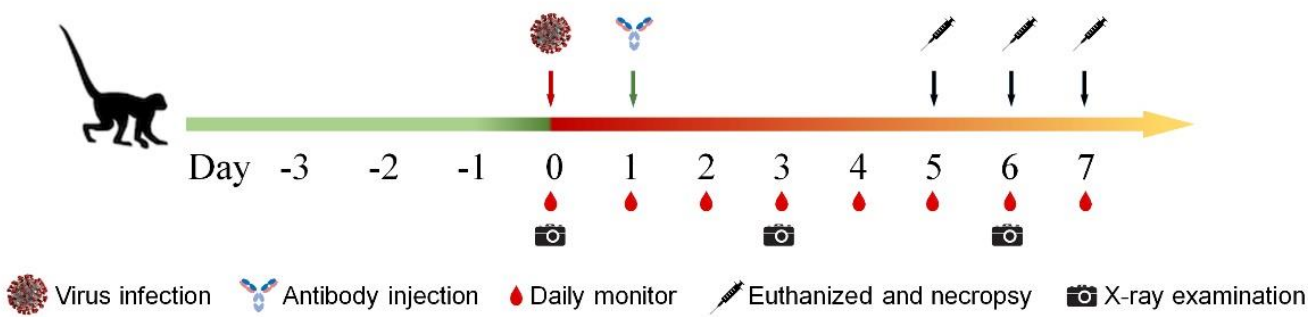

B
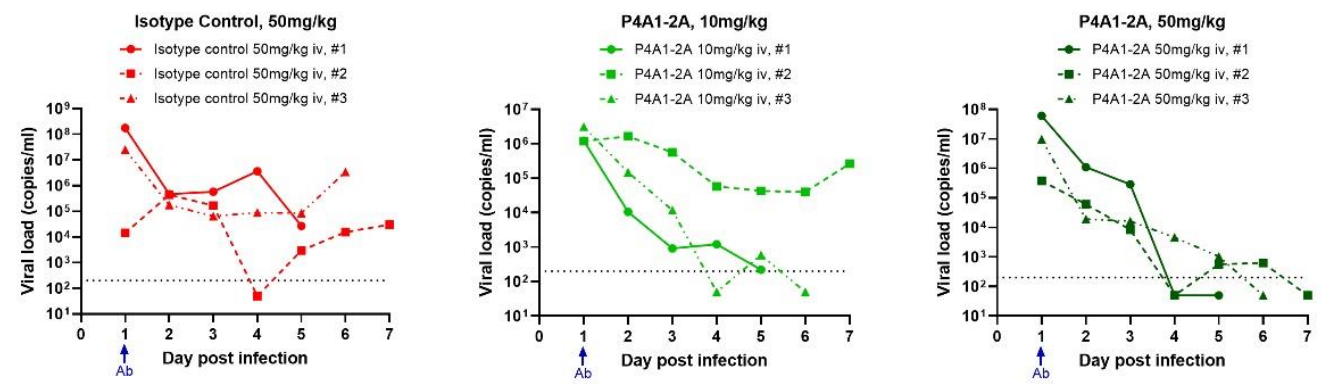

C

$\mathrm{D}$
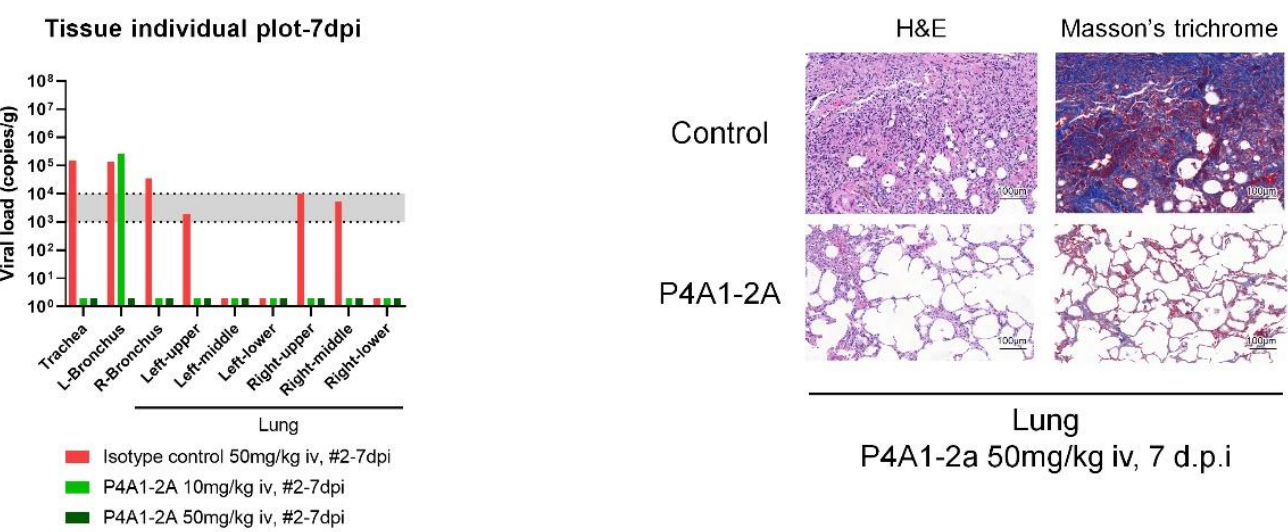

Figure 4. Therapeutic efficacy of in the rhesus macaque model of SARS-CoV-2

356 infection. (A) Experimental design for therapeutic testing of P4A1-2A in the rhesus 357 macaque. (B) Viral load in oropharyngeal swabs tested by RT-qPCR were monitored for 7 358 days. (C) Viral load in the respiratory tissues (including trachea, left and right bronchus, and 359 all six lung lobes) collected at necropsy on 7 days post-infection (d.p.i) were tested by RT360 qPCR. (D) Representative images of histopathology in lung tissue from isotype control or 
361 P4A1-2A $50 \mathrm{mg} / \mathrm{kg}$ treated animals (collected at 7 d.p.i).

362

363

364

365

366

367

368

369

370

371

372 


\section{Methods}

\section{Ethics statements}

3 The study was approved by the Ethics Committee of Xinhua Hospital affiliated to Shanghai

4 Jiao Tong University (approval \#XHEC-C-2020-006-2), Yongjia People's Hospital and

5 Yongjia Center for Disease Control and Prevention in Zhejiang Province. Blood samples

6 were collected from convalescent COVID-19 patients and healthy volunteers with signed

$7 \quad$ informed consent forms according to study protocol approved by IRBs

8 All animal experiments were approved by the Institutional Animal Care and Use Committee

9 (IACUC) of Wuhan Institute of Virology, Chinese Academy of Sciences (Ethics number:

10 WIVA42202001) or Wuxi AppTec (Number: SZ20200529-Monkeys for PK study and

11 SZ20200608-Monkeys for GLP Toxicology study). Rhesus macaque studies were

12 conducted within the animal biosafety level 4 (ABSL- 4) facility in the National Biosafety

13 Laboratory (Wuhan), Chinese Academy of Sciences.

14

15 Study design and participants

16 Convalescent COVID-19 patients were recruited between February 11th, 2020 and March

17 1st, 2020 at Yongjia People's Hospital and Yongjia Center for Disease Control and

18 Prevention in Zhejiang Province, China. All the patients with COVID-19 were clinically

19 diagnosed and laboratory-confirmed. A laboratory-confirmed case of COVID-19 was defined

20 as a positive result on high-throughput sequencing or real-time reverse-transcriptase 
21 polymerase-chain-reaction (RT-PCR) assay of nasal and pharyngeal swab specimens 22 based on the WHO interim guidance (WHO, 2020). The criteria of recovery were normal 23 temperature for at least 3 days, obvious improvement in clinical symptoms, significant 24 absorption of pulmonary inflammation on computer tomography scan and negative tests for 25 SARS-CoV-2 two times in a row with a test interval for at least one day. Individuals tested 26 positive for HIV, HBV, HAV, HCV or syphilis were excluded. All the clinical data were 27 reviewed by a team of physicians from departments of respiratory, intensive care and 28 infectious diseases. Patients without the baseline assessment test were excluded. 23 convalescent COVID-19 patients and one healthy volunteer were included in the study.

30

\section{$31 \quad$ PBMC and serum sample preparation}

32 Blood samples were collected using heparin as anticoagulant 3-4 days after donors were

33 discharged from the hospital and separated into plasma and peripheral blood mononuclear 34 cells (PBMCs) by Ficoll-Hypaque gradient centrifugation. Plasmas and PBMCs in freezing 35 media were stored at $-80^{\circ} \mathrm{C}$. The plasma was heat-inactivated at $56^{\circ} \mathrm{C}$ for $1 \mathrm{~h}$ before use.

\section{Titer measurements by ELISA}

38384 well plates were coated overnight at $4^{\circ} \mathrm{C}$ with PBS containing $1 \mu \mathrm{g} / \mathrm{mL}$ of SARS-CoV-2

39 Spike receptor-binding domain (RBD)-mFc recombinant protein (SinoBiological, catalog \# $4040592-\mathrm{V} 05 \mathrm{H}$ ) or full-length S-his (SinoBiological, catalog \# 40589-V08B1) or S1-mFc

41 (SinoBiological, catalog \# 40591-V05H1) or S1-his (Kactus, catalog \# COV-VM4S1). The 
next day the plate was washed 4 times with washing buffer (PBS and $0.05 \%$ Tween) and

43 then incubated 1 hour at $37^{\circ} \mathrm{C}$ in blocking buffer (PBS with $2 \%$ BSA). After two washes the

44 plate was incubated for 1 hour at $37^{\circ} \mathrm{C}$ with the serum or the positive control ACE2 protein

45 (SinoBiological, catalog \# 10108-H08H). The human serum samples were diluted to 1:100

46 in PBS + 2\% BSA followed by 5-fold serial dilutions. The plates were then washed 4 times

47 and incubated for 1 hour in blocking buffer (PBS with $0.05 \%$ Tween and $1 \%$ BSA) containing

48 diluted (1:5000) secondary antibody (HRP labelled mouse anti-human IgG Fc antibody,

49 Thermo Fisher, catalog \# 05-4220, clone name: HP6017) for 1 hour at room temperature.

50 Following this the plate was washed again 4 times and developed in TMB substrate

51 (Biolegend, catalog \# 421101) for 5 min before stopping the reaction with the stop solution

52 (Solarbio, catalog \# C1058).

53

54 Flow cytometry for B cell immune profiling

55 PBMCs was thawed at $37^{\circ} \mathrm{C}$ and then centrifuged at $450 \mathrm{x} \mathrm{g}$ for $8 \mathrm{~min}$. The supernatant was

56 discarded, and the cells resuspended in $200 \mu \mathrm{L}$ of DMEM (Gibco, catalog \# 11995-065).

57 Following the addition of $1 \mu \mathrm{L}$ of Dnase I (Qiagen, catalog \# 79254), cells were incubated for

583 min and spun down again. The pellet was resuspended in $20 \mu \mathrm{L}$ of FcR Blocking Reagent

59 (Miltenyi Biotec, catalog \# 130-059-901), incubated for 10 mins and centrifuged. The cells

60 were suspended in $200 \mu \mathrm{L}$ PBS. $3 \mu \mathrm{L}$ (1:70 dilution) of anti-CD19 (FITC labeled, eBioscience,

61 catalog \# 11-0199-42, clone name: HIB19), anti-CD27 (APC labeled, eBiosciences, catalog

62 \# 17-0279-42, clone name: O323), anti-CD38 (PE labeled, eBioscience, catalog \# 12-0388- 
42, clone name: HB7) or its isotype PE Mouse isotype control (BioLegend, catalog \# 400114,

64 clone name: MOPC-21) and FITC labeled mouse IgG1 isotype control (ebioscience, catalog

65 \# 11-4714-41, clone name: P3.6.2.8.1) and APC labeled mouse IgG1 isotype control (BD

66 Biosciences, catalog\#550854, clone name: MOPC-21) was then added, and incubated for

$6730 \mathrm{~min}$ at room temperature. Following centrifugation, cells were resuspended in a $100 \mu \mathrm{L}$

68 of 4\% Paraformaldehyde fix solution (PFA; Beyotime, catalog \# P0099-500ml). After 10 min

69 the cells were washed twice and finally resuspended in PBS and analyzed using a Cytoflex

70 (Beckman Coulter) flow cytometer. The median fluorescence intensity (MFI) was calculated

71 with FlowJo (version 10.6.0).

72

73

Patient sample information for FACS

\begin{tabular}{|l|l|l|l|l|}
\hline & Patient \# & Cell Number & Viability (\%) Gender \\
\hline PBMC & Patient 4 & $2.54 \times 10^{5} / \mathrm{mL}, 200 \mu \mathrm{L}$ & 84 & Female \\
\hline PBMC & Patient 20 & $7.1 \times 10^{5} / \mathrm{mL}, 200 \mu \mathrm{L}$ & 90 & Male \\
\hline PBMC & Healthy donor & $7.0 \times 10^{5} / \mathrm{mL}, 200 \mu \mathrm{L}$ & 92 & Male \\
\hline
\end{tabular}

74

75 Isolation of S protein specific B cells

76 Avi tag and His tag SARS-CoV-2 S protein was expressed in human embryonic kidney cell

77 293-F (ThermoFisher, catalog\# R79007. The cell line was not authenticated.) which was

78 tested negative for mycoplasma contamination. The S protein was purified using HISTRAP

79 HP column (GE, catalog \# 17-5248-01) and biotinylated using Biotin-Protein Ligase

80 (GeneCopoeia, catalog \# BI001). The B cells were stained with biotinylated S protein and

81 incubated at $4^{\circ} \mathrm{C}$ for 1 hour. After incubation, the cells were washed three times with PBS. 
82 Subsequently, the cells were labelled with Streptavidin microbeads (Miltenyi Biotec, catalog

83 \# 130-048-101) at $4^{\circ} \mathrm{C}$ for 1 hour. After the incubation, the cell suspension was loaded onto

84 a MACS column which is placed on a magnetic field of a MACS separator. The column was

85 washed three times so the magnetically labelled B cells were retained in the column and

86 unlabeled cells passed through. After removing from the MACS separator, the magnetically

87 labelled B cells were eluted. The isolated B cells were counted by using $0.4 \%(w / v)$ Trypan

88 blue stain.

89

\section{$90 \quad$ Single-cell BCR sequencing}

91 Enriched S protein specific B cells were individually co-compartmentalized in droplets with

92 single barcoded hydrogel beads and lysis and reverse transcription reagents using a

93 microfluidic device as described15. Droplets of $\sim 1 \mathrm{~nL}$ volume were formed at $250 \mathrm{~s}-1$. The

94 droplets were collected in a 1.5mL tube containing HFE-7500 (3MTM, catalog \# Novec 7500)

95 and $0.1 \%$ surfactant (RanBio, catalog \# 008-FluoroSurfactant), UV photo-cleaved for 90

96 seconds (OmniCure ac475-365) and incubated at $50^{\circ} \mathrm{C}$ for cell lysis and cDNA synthesis.

97 Reverse transcription (RT) of $\mathrm{VH}$ and $\mathrm{VL}$ mRNAs from single B cells took place in droplet

98 using barcoded primers carrying the T7-SBS12 sequences followed by barcode and gene-

99 specific primer sequences complementary to heavy chain $\mathrm{J}$ genes and light chain constant 100 region sequences.

101 The emulsion containing the barcoded cDNA was broken by adding one volume of $1021 \mathrm{H}, 1 \mathrm{H}, 2 \mathrm{H}, 2 \mathrm{H}-$ Perfluoro-1-octanol (Sigma-Aldrich, catalog \# 370533-25G) after the droplet 
103 RT reaction finished. The pooled, barcoded cDNAs were purified with Agencourt RNA 104 CleanUp beads (Beckman, catalog \# A63987) at a 1:1 ratio (vol/vol) twice and eluted in 60 $105 \mu \mathrm{L}$ DNase- and RNase-free $\mathrm{H}_{2} \mathrm{O}$. The sequencing library was generated by two-step nested 106 PCR using GoTaq Polymerase (Promega, catalog\#M7406). In the first PCR, forward primers 107 were priming on the $\mathrm{T} 7$ and with reverse primers priming on the $\mathrm{VH}, \mathrm{V} \lambda$ and $\mathrm{Vk}$ leader and 108 framework 1 sequenced of the $\mathrm{V}$ genes. In the second PCR, the forward primer appends 109 the Illumina P7 and Illumina index sequences by priming on the SBS12 sequence and the 110 reverse primer appends the Illumina P5 and SBS3 sequence. The approximately 550 bp 111 final PCR products were extracted by agarose gel electrophoresis (Qiagen, catalog\#28606). 112 The constructed NGS libraries were sequenced using Illumina MiSeq PE300 which allows 113 sequencing of the entire $\mathrm{VH}$ and VL domain as well as the barcode sequence (GeneScript 114 sequencing service supplier) with data varying from 6-12 million reads per samples. The 115 resulting FASTQ data was analyzed by a bioinformatics pipeline enable trimming, merging, 116 barcode extraction and clustering as described(1). Briefly, paired-end reads were first 117 trimmed at the 3 ' end to remove low quality score bases then merged using the program 118 FLASH requiring at least 10bp overlap. The barcodes were extracted from merged reads 119 followed by clustering requiring the DNA sharing at least $93 \%$ identify. The consensus 120 sequence was created from clusters by aligning up to 200 sequencings using 121 ClustalO(version1.2), and each antibody sequence was characterized for immunoglobulin 122 content using VDJFasta(version 2.0). We also applied a minimum number of reads 10 for $123 \mathrm{VH}$ and $\mathrm{VL}$. $\mathrm{VH}-\mathrm{VL}$ pairing was carried out by identifying the most abundant $\mathrm{VH}$ and $\mathrm{VL}$ 
consensus sequence (by the number of reads that contributed to that in each barcode 125 cluster).

Production of recombinant antibody

128 The DNA of P4A1, P20A2 and P20A3 variable regions of the heavy and light chains were synthesized and cloned into expression plasmids containing the human IgG1 heavy chain 130 and kappa light chain constant regions. The antibodies were expressed in ExpiCHO cell for 1318 days after the co-transfection of both heavy and light chain expression plasmids. P4A12A was produced by cloning of P4A1 variable regions into expression plasmid containing human IgG4 heavy chain with Fc modifications and expressed in CHO.K1 cells. Antibodies were purified from cell culture supernatants using Protein-A affinity chromatography.

\section{Antibody binding and competition with receptor ACE2}

137 The binding affinity of antibodies to $S$ protein was analyzed by ELISA. 384 well plate 138 (Corning, catalog \# 3700), was coated overnight at $4^{\circ} \mathrm{C}$ with PBS containing $30 \mu \mathrm{L} 20 \mathrm{nM}$ of 139 the SARS-CoV-2 Spike S1+S2 ECD, his Tag protein (SinoBiological, catalog \# 40589140 V08B1). The next day the plate was washed 5 times with washing buffer (PBS and $0.05 \%$

141 Tween) and then incubated 1 hour at room temperature in blocking buffer (PBS with $2 \%$ 142 BSA). After 5 washes the plate was incubated with a serial dilution of purified antibodies for 1431 hour at room temperature. The plates were then washed 5 times and incubated for 1 hour 144 in blocking buffer (PBS with $0.05 \%$ Tween and 1\% BSA) containing Mouse anti-Human IgG 
145 Fc HRP labelled (Thermo Fisher, catalog \# 05-4220, clone name: HP6017, dilution 1:5000)

146 for 1 hour at room temperature. The plate was then washed again 5 times and incubated 147 with TMB substrate (Biolegend, catalog \# 421101) for 5 min before adding the stop solution 148 (Solarbio, catalog \# C1058). The OD values at 450nm wavelength were determined using 149 Thermo MultiSkan or MD SpectraMax i3X, data were analyzed with GraphPad Prism 150 (version 8.0.1).

151 The blocking S1 binding with Vero-E6 cell surface ACE2 receptor was performed using flow 152 cytometry analysis. Vero-E6 cell line (ATCC, CRL-1587) was validated by CoBIOER 153 (http://www.cobioer.com/) and tested negative for mycoplasma contamination. $10 \mathrm{nM}$ SARS154 CoV-2 spike S1, mFc tag protein (SinoBiological, catalog \# 40591-V05H1) was incubated 155 with a serial dilution of purified antibodies in duplicates at room temperature for 1 hour and 156 then added to Vero E6 cells (105 cells per well). Rabbit anti-mouse IgG Fc-AF647 (Jackson 157 ImmunoResearch, catalog \# 315-606-046, 1:800 dilution) was then added before final wash 158 and data acquisition with a Cytoflex flow cytometer (Beckman) and data analysis using 159 FlowJo software (version 10.6.0). Non-linear regression was used to calculate the $\mathrm{IC}_{50}$ of 160 the evaluated antibodies with GraphPad Prism (version 8.0.1).

162 Antibody neutralization activity against pseudovirus

163 Huh-7 cell line, which expresses ACE2 receptor, was infected with pseudo-virus expressing 164 the full length of SARS-CoV-2 Spike protein and luciferase reporter gene in the presence 165 and absence of serial dilutions of testing antibodies. Viral entry to the cells was quantified 
using BriteliteTM plus Reporter Gene Assay System. In separate studies, pseudoviruses

167 encoding wild type or different mutant S protein (GenScript, SARS-CoV-2/Wild-type (WT), SARS-CoV-2/D614G, SARS-CoV-2/V367F, SARS-CoV-2/W436R and SARS-CoV-2/D364Y) were incubated with a serial dilution of purified antibodies (starting from $100 \mu \mathrm{g} / \mathrm{ml}, 3$ folds

170 dilution for 8 points) at room temperature for 1 hour. The mixture was then added to ACE2 171 overexpressing HEK293 cells $\left(2 \times 10^{4}\right.$ per well) cultured in DMEM containing $10 \%$ FBS in 172 triplicate. Following infection at $37^{\circ} \mathrm{C} 5 \% \mathrm{CO}_{2}$ for 48 hours, luciferase activity was determined 173 using the Promega Bio-Glo luciferase assay (Promega, catalog \# G7491) system. The dose174 response curves were plotted with the relative luminescence unit against the sample 175 concentration. Non-linear regression was used to calculate $\mathrm{IC}_{50}$ using GraphPad Prism 6.

\section{Live virus assay}

178 Microneutralization assays towards live virus were performed as previously described(2) 179 with slight modifications. The Vero E6 cell line (ATCC; CRL-1586, Lot\#: 60526234; this clone 180 was not authenticated) was tested negative for mycoplasma contamination using a 181 commercial EZ-PCRTM Mycoplasma Test kit (Biological Industries, Beit-Haemek, Israel; 20182 700-20; Lot\#: 1251719) (data not shown). Briefly, SARS-CoV-2 (strain 183 BetaCoV/Wuhan/WIV04/2019 preserved in National Virus Resource Center under the 184 accession number: IVCAS 6.7512) was mixed with the equivalent volume of culture medium 185 containing serially diluted antibodies and incubated at $37^{\circ} \mathrm{C}$ for 1 hour. Then, Vero E6 cells 186 seeded in 96-well plates were incubated with a pre-incubated mixture containing virus at 
$10050 \%$ tissue-culture infectious doses ( $\left.\mathrm{TCID}_{50}\right)$ per well and diluted antibodies and were

188 further sustained at $37^{\circ} \mathrm{C}$ for 48 hours. Subsequently, cells were fixed with $4 \%$ 189 paraformaldehyde diluted in PBS for 15 minutes and penetrated by $0.25 \%$ Triton-X 100 . 190 After three washes with PBS, cells were blocked at $37^{\circ} \mathrm{C}$ for 1 hour using PBS containing $5 \%$ 191 BSA, then incubated with in-house prepared anti-SARS-CoV-2 nucleocapsid protein (NP) 192 rabbit serum produced internally at 1:1000 dilution as primary antibody and Goat Anti-Rabbit 193 IgG H\&L (Alexa Fluor® 488) (1:500 dilution, Abcam, Cambridge, UK; ab150077; Lot\#: 194 GR3244688-2) as the secondary antibody. Cell nuclei were stained using Hoechst 33258 195 (Beyotime, Shanghai, China; C1018). Images were taken, and numbers of nuclei and cells 196 infected with viruses were counted, respectively, using an Operetta CLSTM system 197 (PerkinElmer, Waltham, USA). Inhibition was calculated by (total nuclei-infected cells)/ total 198 nuclei $\times 100 \%$. Fifty percent neutralization dose $\left(N_{50}\right)$ were calculated with GraphPad 199 Prism 8.0.

201 Surface plasmon resonance analysis of wild-type or mutant RBD/S1 binding.

202 SPR experiments were performed using Biacore T200 system (GE Healthcare). In brief, 203 experiments were performed at $25^{\circ} \mathrm{C}$ in HBS-EP+ buffer. The antibody was immobilized onto 204 a protein A sensor chip (GE healthcare, catalog\#29139131-AA). Serially diluted SARS-CoV2052 RBD (WT, AcroBiosystems, catalog\#SPD-C52H3), RBD (N354D/D364Y, AcroBiosystems, 206 catalog\#SPD-S52H3), RBD (R408I, AcroBiosystems, catalog\#SPD-S52H8), RBD (W436R, 207 AcroBiosystems, catalog\#SPD-S52H7), RBD (V367F, AcroBiosystems, catalog\#SPD- 
S52H4) or SARS-CoV-2 spike S1 domain (D614G, Sino Biological, catalog\#40591-V08H3) were injected through flow cells for 60 s of association followed by a 150 s dissociation phase

210 at a flow rate of $30 \mu \mathrm{L} \min -1$. Prior to the next cycle, the sensor surface was regenerated 211 with Glycine- $\mathrm{HCl}(\mathrm{pH}$ 1.5) for $30 \mathrm{~s}$ at a flow rate of $30 \mu \mathrm{L}$ min-1. $\mathrm{KD}$ values were calculated 212 using the 1:1 binding kinetics model.

\section{Protein expression and purification}

215 The codon-optimized wild type cDNA of SARS-CoV-2 RBD (residues 333-530) was 216 synthesized. The SARS-CoV-2 RBD with a C-terminal $8 \times$ His tag for purification was cloned 217 into pAcgp67 vector with BamH I and Not I restriction sites using the cloning primers. The 218 sequences of the primers 5'219 TCTCCTACATCTACGCCGACGGATCCACCAACCTCTGCCCTTTCGGT-3' (forward), 5'220 TGGTGATGGTGGTGATGATGTGCGGCCGCACTCTTCTTTGGCCCGCATA -3' (reverse). 221 The accuracy of the inserts was verified by sequencing.

222 The SARS-CoV-2 RBD was expressed using the Bac-to-Bac baculovirus system. The 223 construct was transformed into bacterial DH5a component cells, and the extracted bacmid 224 was then transfected into Sf9 cells using Cellfectin II Reagent (Invitrogen). The low-titer 225 viruses were harvested and then amplified to generate high-titer virus stock. The viruses 226 and Endo $\mathrm{H}$, Kifunensine were co-infected $\mathrm{Hi} 5$ cells at a density of $2 \times 10^{6} \mathrm{cells} / \mathrm{ml}$. The 227 supernatant of cell culture containing the secreted removal of glycosylated RBD was 228 harvested $72 \mathrm{~h}$ after infection, concentrated and RBD was captured by Ni-NTA resin (GE 
229 Healthcare). The resin was washed 5-6 times with $30 \mathrm{~mL}$ of wash buffer (25 mM Tris, 150 $230 \mathrm{mM} \mathrm{NaCl}, 40 \mathrm{mM}$ imidazole, $\mathrm{pH}$ 7.5), the target protein was eluted with elution buffer 231 containing $25 \mathrm{mM}$ Tris, $150 \mathrm{mM} \mathrm{NaCl}, 500 \mathrm{mM}$ imidazole, $\mathrm{pH}$ 7.5. The protein was further 232 purified on a Superdex S75 (GE Healthcare) column equilibrated with $25 \mathrm{mM}$ Tris, $150 \mathrm{mM}$ $233 \mathrm{NaCl}, \mathrm{pH}$ 7.5. SDS-PAGE analysis revealed over $95 \%$ purity of the final purified recombinant 234 protein. Fractions from the single major peak were pooled and concentrated to $15 \mathrm{mg} / \mathrm{mL}$ 235 RBD were collected.

\section{Crystallization}

238 The SARS-CoV-2 RBD protein and P4A1-Fab fragment were mixed at a molar ratio of 1.5:1. 239 The mixture was incubated on ice for $1 \mathrm{~h}$ and further purified by Superdex S75 (GE 240 Healthcare). $7 \mathrm{mg} / \mathrm{mL}$ and $10 \mathrm{mg} / \mathrm{mL}$ of SARS-CoV-2 RBD/Fab proteins were used for 241 crystal screening by vapour-diffusion sitting-drop method at $16^{\circ} \mathrm{C}$, including the Index, 242 Crystal Screen, PEG/lon, SaltRX from Hampton Research and wizard I -IV from Emerald 243 BioSystems.

244 The rode-like crystals appeared after two days at the mother liquid containing $20 \% \mathrm{w} / \mathrm{v}$ PEG $2453350,0.2 \mathrm{M}$ potassium citrate tribasic. Further optimization with additive and hanging-drop 246 vapour-diffusion method was performed, the final optimized diffraction crystals at the mother 247 liquid containing 20\% w/v PEG 3350, $0.2 \mathrm{M}$ potassium citrate tribasic by the hanging-drop 248 vapour-diffusion method. Crystals were dehydrated and cryo-protected in 4M Sodium 249 formate solution and cooled in a dry nitrogen stream at $100 \mathrm{~K}$ for $\mathrm{X}$-ray data collection. 
251 X-ray data collection, processing, and structure determination

252 Diffraction data were collected at the Shanghai Synchrotron Radiation Facility (SSRF) 253 BL17U1 (wavelength, $0.97915 \AA$ ) at $100 \mathrm{~K}$. All data sets were processed using the HKL3000 254 package(3). Structures were solved by molecular replacement using PHASER(4) with the 255 SARS-CoV-2 RBD structure (PDB ID: 6MOJ) (5) and the structures of the Fab fragment 256 available in the PDB with the highest sequence identities. The initial model was built into the 257 modified experimental electron density using COOT (6) and further refined in PHENIX(7). 258 Model geometry was verified using the program MolProbity. Structural figures were drawn 259 using the program $\operatorname{PyMOL}(8)$ (http://www.pymol.org). Epitope and paratope residues, as 260 well as their interactions, were identified by accessing PISA 261 (http://www.ebi.ac.uk/pdbe/prot_int/pistart.html) at the European Bioinformatics Institute.

263 Binding affinity to FcyRs and C1q

264 The binding affinity of P4A1-2A as well as its IgG1 form for different human FcyRs, FcRn 265 was tested by SPR using Biacore 8K (GE Healthcare). FcyRI, FcyRIla (H131), FcyRllb, 266 FcyRIIla (V158) (all from AcrobioSystems) were captured on the activated CM5 sensor chips, 267 followed by flow-through of 10 concentrations of serial diluted P4A1 (IgG1) or P4A1-2A 268 (IgG4) antibodies. For FcRn binding, P4A1 or P4A1-2A were captured on the activated CM5 269 sensor chips followed by flow-through of 10 concentrations of serial diluted FcRn 270 (AcrobioSystems). The sensor chips were regenerated by flow-through of $10 \mathrm{mM}$ glycine 
$271(\mathrm{pH} 1.5)$

272 Antibody binding to $\mathrm{C} 1 \mathrm{q}$ was tested by ELISA, with P4A1 or P4A1-2A antibodies to coat the 273 plate overnight at $4^{\circ} \mathrm{C}$, followed by incubation with 11 concentration of half-log titrated human

274 C1q (Complement Technology), then incubation with secondary antibody Sheep anti-human 275 C1q Ab-HRP (Complement Technology), before TMB substrate was added and absorbance 276 at $450 \mathrm{~nm}$ was determined using a SpectraMax Plus384 microplate reader.

\section{RT-PCR}

279 Total RNA was extracted from organs with an RNeasy Mini Kit (Qiagen, USA) and 280 PrimerScript RT Reagent Kit (TaKaRa, Japan). The forward and reverse primers targeting 281 the SARS-CoV-2 NP gene for RT-PCR were 5'-GGGGAACTTCTCCTGCTAGAAT-3' and 5'282 CAGACATTTTGCTCTCAAGCTG-3', respectively. RT-PCR was performed under the 283 following reaction conditions: $42^{\circ} \mathrm{C}$ for $5 \mathrm{~min}, 95^{\circ} \mathrm{C}$ for $10 \mathrm{~s}$, and 40 cycles of $95^{\circ} \mathrm{C}$ for $10 \mathrm{~s}$ 284 and $60^{\circ} \mathrm{C}$ for $30 \mathrm{~s}$.

\section{Pharmacokinetic (PK) analysis}

287 Six (6, 3/sex) naïve cynomolgus monkeys were enrolled in the study and administered with $288 \mathrm{P} 4 \mathrm{~A} 1-2 \mathrm{~A}$ at $10 \mathrm{mg} / \mathrm{kg}$ by single intravenous infusion. Blood samples PK analysis were 289 collected at pre-dose, and different time-points after the treatment. Serum concentrations of 290 P4A1-2A were determined by ELISA. 


\section{Safety evaluation}

293 Cynomolgus monkeys (3-5 years old, 2.2 to $3.6 \mathrm{~kg}$ for females and 2.3 to $5.2 \mathrm{~kg}$ for males, 294 sourced from GuangDong Blooming-Spring Biological Technology Development Co., Ltd.) 295 were randomly assigned to 3 groups of 5/sex/group and given P4A $1-2 \mathrm{~A}$ at 0,50 , or 300 $296 \mathrm{mg} / \mathrm{kg} /$ dose on Day 1 and Day 8 by intravenous (IV) infusion. Animals were monitored daily 297 with blood samples collected at different time-point for various testing. Animals were 298 euthanized on Day 15 to examine thoroughly for any potential toxicity.

300 In vivo efficacy in SARS-CoV-2 infection model in rhesus monkey

301 Nine rhesus monkeys (RMs, three males, six females; 6-7 years of age, 5.3-7.3 kg, sourced 302 from Hubei Tianqin Biotech Company), were inoculated with SARS-CoV-2 virus 303 (BetaCoV/Wuhan/WIV04/2019)(2) at $1 \times 10^{5}$ TCID 50 intratracheally under anesthesia on 304 Day 0. Animals were randomized into 3 groups (1 male and 2 females in each group) and 305 receive a single intravenous (i.v.) treatment of Isotype control W332-1.80.12.xAb.hlgG4 306 (sourced from Wuxi Biologics, Co. Ltd.) at $50 \mathrm{mg} / \mathrm{kg}$, or P4A1-2A at 10 or $50 \mathrm{mg} / \mathrm{kg}$, 307 respectively, one day after viral infection. The RMs were observed twice daily with detailed 308 recording of clinical signs. Swab samples of the oropharyngeal, nasal turbinate, and rectal 309 regions, as well as whole blood (in K2EDTA tubes), were collected at 0-7 days post-infection 310 (d.p.i.). To confirm the pathogenesis and injury in the respiratory system, one animal from 311 each treatment groups were sacrificed at 5-7 d.p.i., respectively. The trachea, right bronchus, 312 left bronchus, all six lung lobes and other tissue organs were collected on the day of 
313 euthanization for various pathological, virological analyses. Viral loads in swabs and tissues

314 were determined and pathological examination was conducted as previously described (9). 315

316 Data availability

317 Protein coordinate and structure factors are deposited in the RCSB Protein Data Bank 318 under ID 7CJF. All data is available in the main text or the supplementary materials. 
A

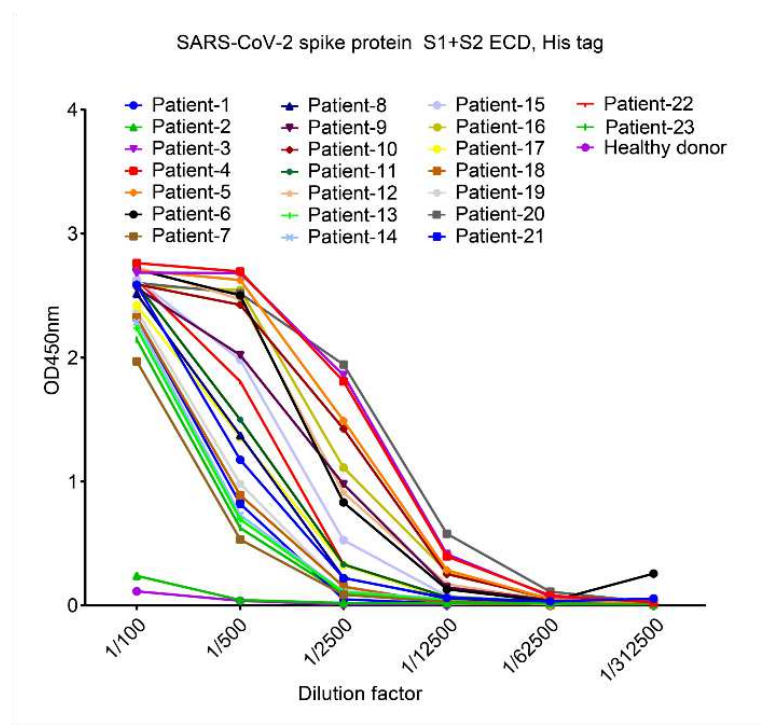

C

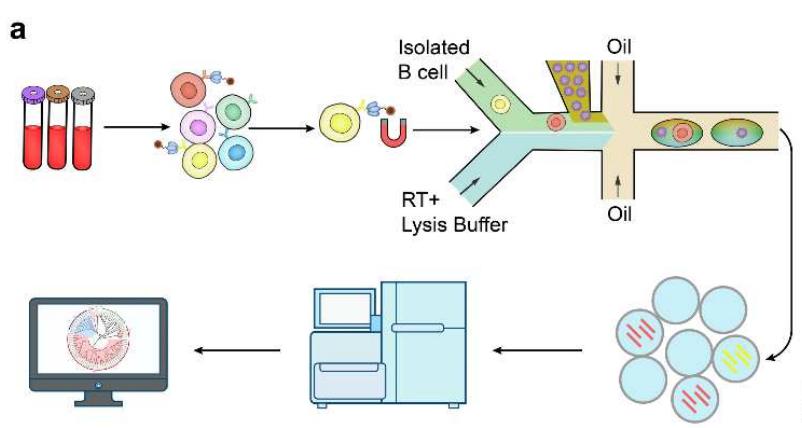

B

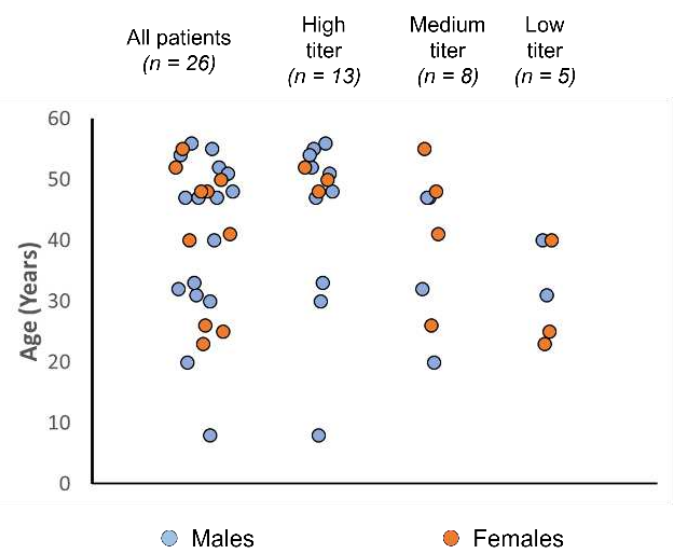

D

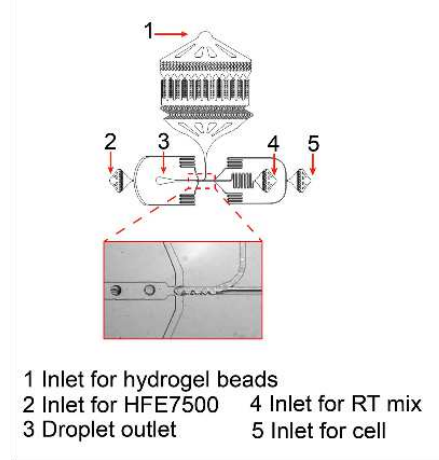

323 Fig.S1. Analyses of plasma responses to SARS-CoV-2 proteins and antibody 324 identification from convalescent patients using single cell sequencing.

325 (A) Serums from 23 convalescent patients and one healthy donor were analyzed for their 326 binding abilities to the SARS-CoV-2 spike protein using ELISA. (B) Classification of patient 327 samples into high $(>2500)$, medium $(500-2500)$, and low $(<500)$ titer categories. (C) 
328 Schematic diagram of the antibody identification from convalescent patients using single cell 329 sequencing. SARS-CoV-2 S protein binding B cells were isolated from PBMC of 330 convalescent patients with magnetic beads that conjugated with biotinylated $\mathrm{S}$ protein as 331 probes. The isolated cells were individually co-compartmentalized in droplets along with 332 Iysis buffer, reverse transcriptase and one hydrogel bead. Each hydrogel bead carried VH 333 and VL specific oligos tagged with a unique barcode. The resulting cDNAs from one cell 334 carried an identical barcode. The barcoded cDNAs were sequenced to identify cognate 335 heavy and light chain pairs. (D) The design of the microfluidics chip for co336 compartmentalization of single cells and single hydrogel bead in droplets. 337 
A

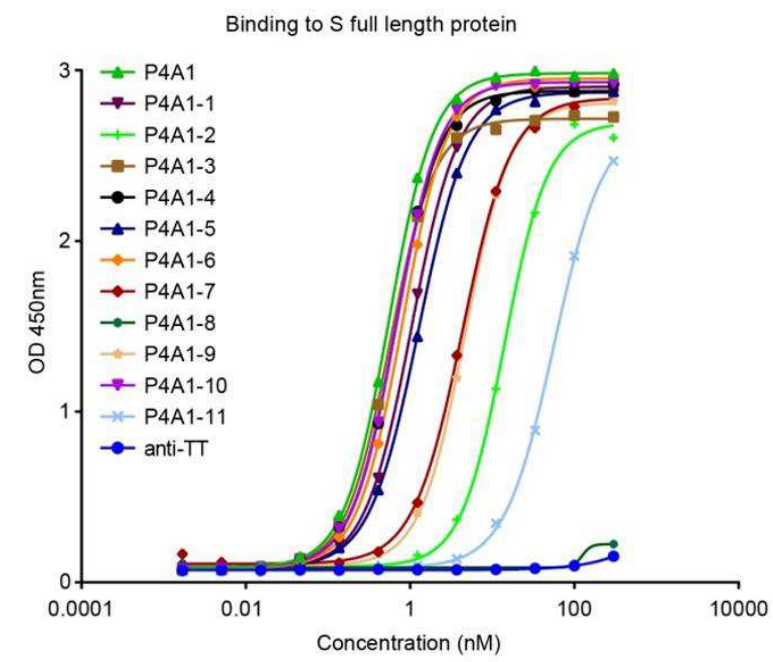

B

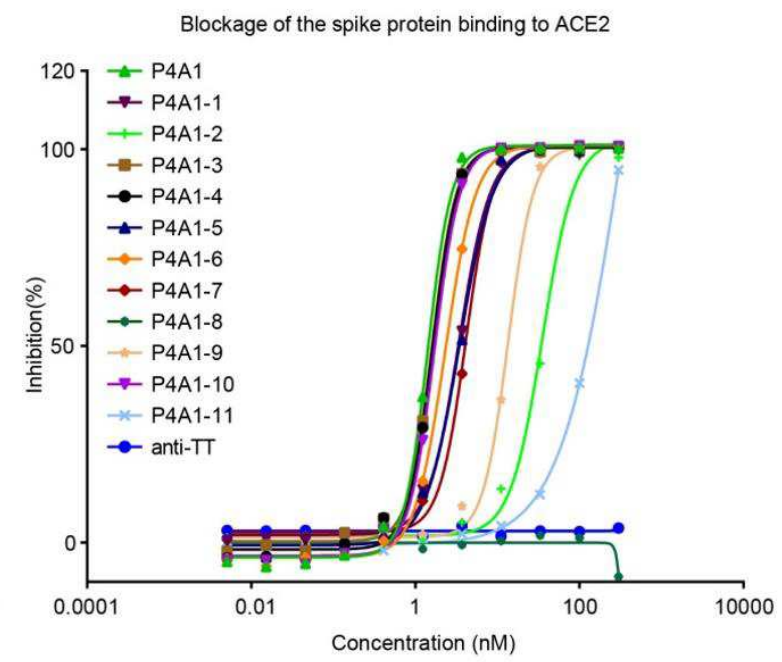

C

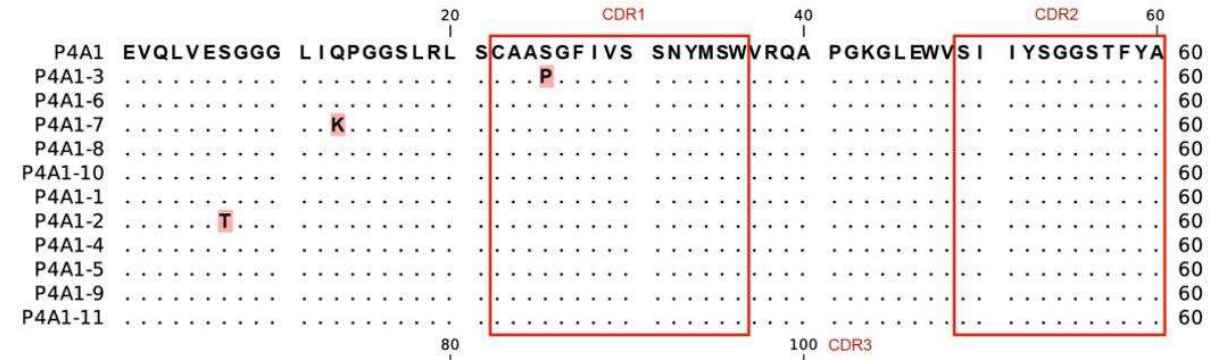

P4A1 DSVKGRFTIS RDNSKNTLYL QMNSLRVEDT AVYYCARDLQ ELGSLDWGQ GTLVTVSS 118

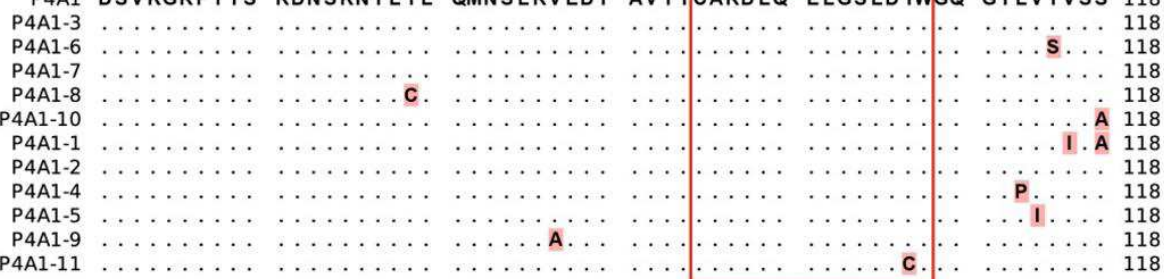

340 Fig.S2. Identification and characterization of somatic variants of antibody P4A1.

341 Eleven heavy chain sequences closely related to P4A1 were bioinformatically identified from

342 NGS results. These P4A1-class heavy chains were reconstituted with the light chain from

343 P4A1 and numbered P4A1-1 to P4A1-11. (A) Binding of the antibodies to the full-length S

344 protein was evaluated by ELISA. (B) Blocking the binding of S1 protein to Vero E6 cell line 
345 by antibodies was evaluated by flow cytometry. (C) The amino acid sequences of the P4A1346 class antibodies were aligned.

347

348 
A

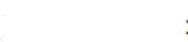

P4A1

CC12. 1

CC12. 3

B38

IGHV3-53
1 VLVESGG I VOLVESGGGI QVQLVESGGG I EVQLVESGGGI VQLVESGGGI
HCDR1

20

\section{HCDR2} $50 \quad 60$

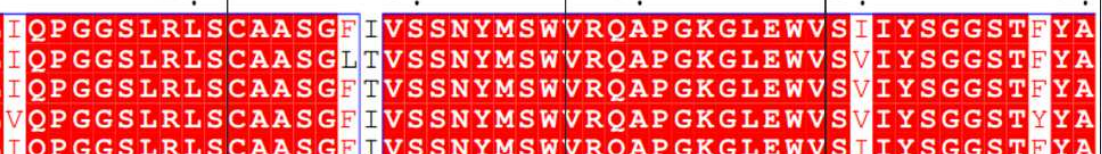

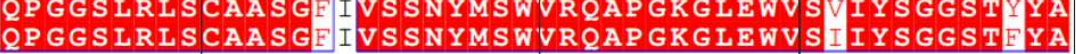

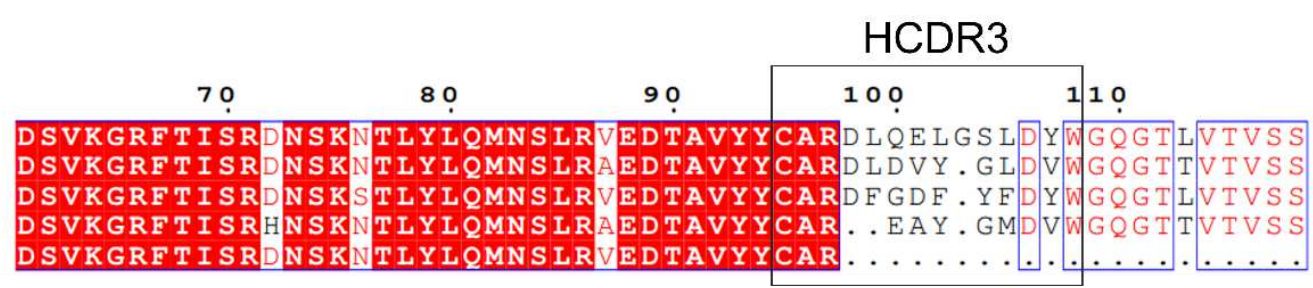

B

P4A1
CC12.1
CC12.3
B38
IGHV3-53

IGHV3-53

TR

T L Y LOMNS L

EDTAVYYCAR
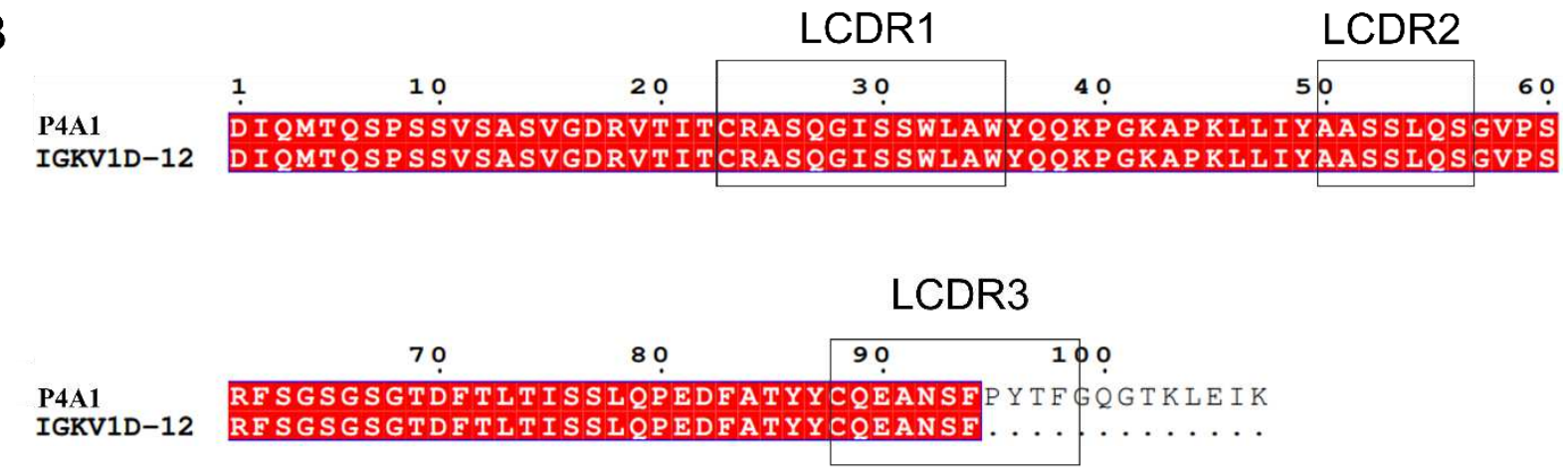

C

Fig.S3. Sequence alignment of P4A1 to other reported antibodies from the IGHV3-53

352 germline. (A) Alignment of the heavy chain variable domain sequence of P4A1 with CC12.1, CC12.3, B38 and the germline IGHV3-53 sequence. (B) Alignment of the light chain variable

354 domain sequence of P4A1 with the germline IGKV1D-12 sequence. (C) Germline usage 355 comparison of the heavy chain and light chain discussed in this paper. 
A

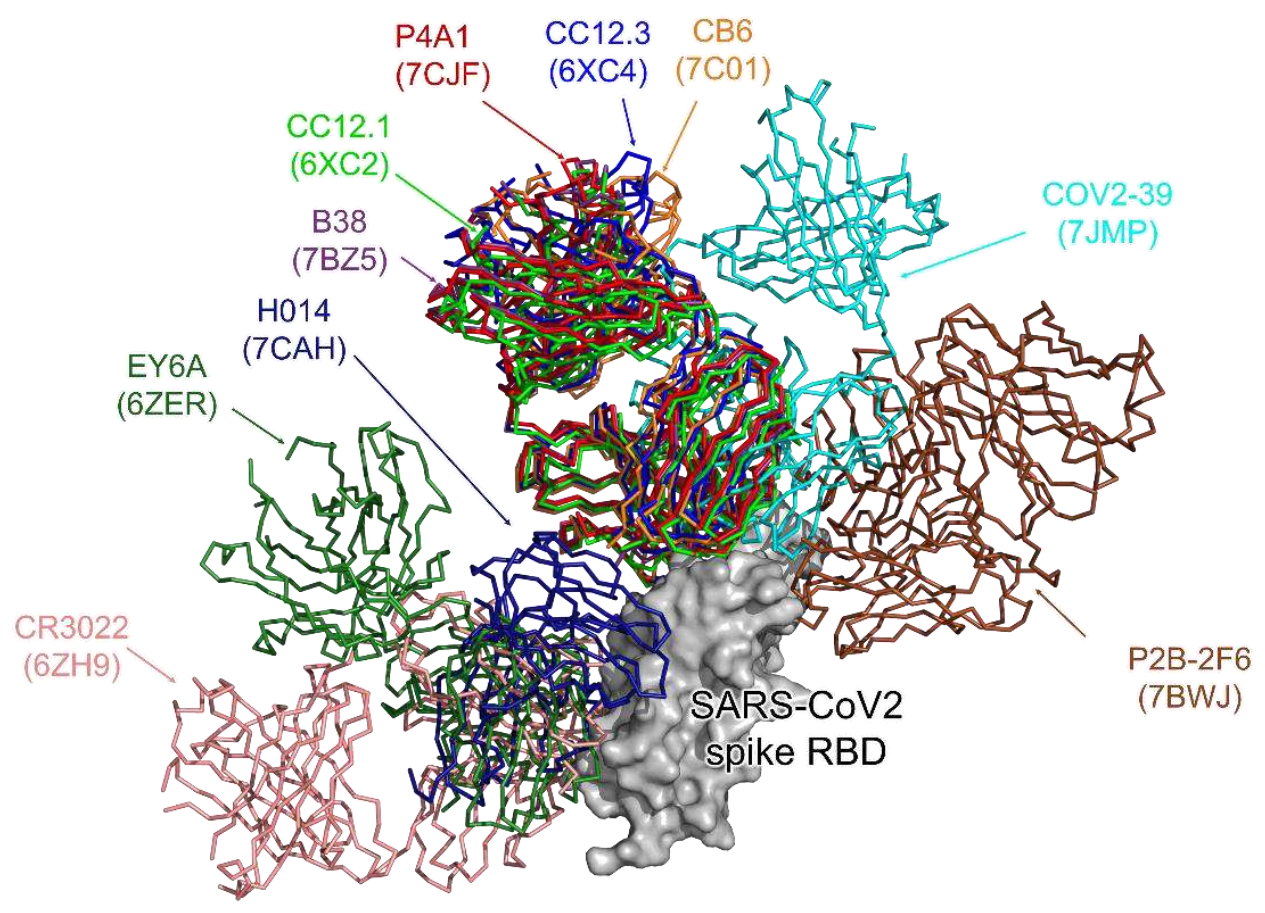

B
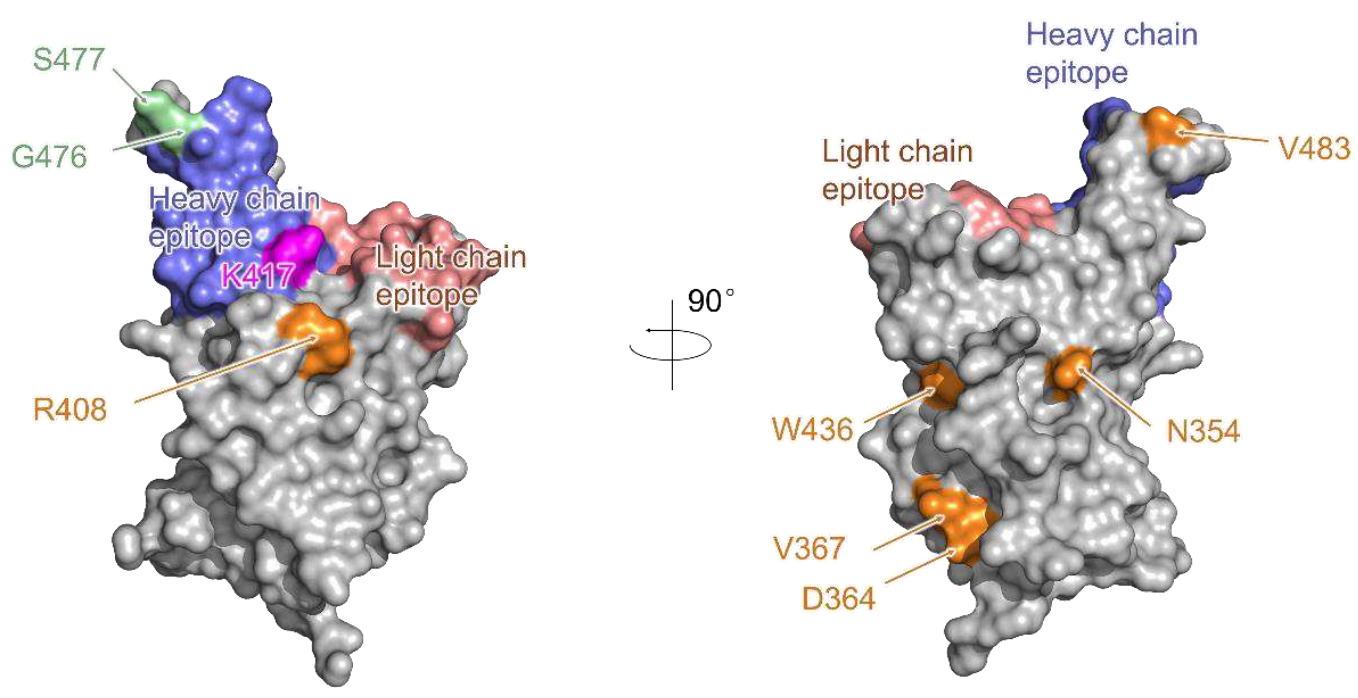

358 Fig.S4. Structural comparison of the binding mode among several reported RBDspecific neutralizing antibodies from various germlines. (A) Superimpose of P4A1 (red, 360 PDB 7CJF), CC12.1 (green, PDB 6XC2), CC12.3 (blue, PDB 6XC4), B38 (purple, PDB 361 7BZ5), CB6 (orange, PDB 7C01), COV2-39 (cyan, PDB 7JMP), H014 (deep blue, PDB 
362 7CAH), EY6A (forest green, PDB 6ZER), CR3022 (salmon red, PDB 6ZH9), P2B-2F6 363 (brown, PDB 7BWJ), to SARS-CoV-2 spike glycoprotein RBD (gray). (B) Surface 364 representation of several clinical isolates with Spike RBD mutations. The SARS-CoV-2 RBD 365 is colored in gray and displayed in surface representation. The epitope of P4A1 heavy chain 366 (slate blue), light chain (salmon red), residue K417 (pink) are displayed and colored as 367 Figure2. The clinic mutations G476 and S477, which located at the edge of the P4A1 epitope 368 are colored in pale green. The clinic mutations N354, D364, V367, R408, W436, and V483, 369 which are adjacent to the epitope residues or on the opposite side of the P4A1 epitope, are 370 colored in orange.

371 

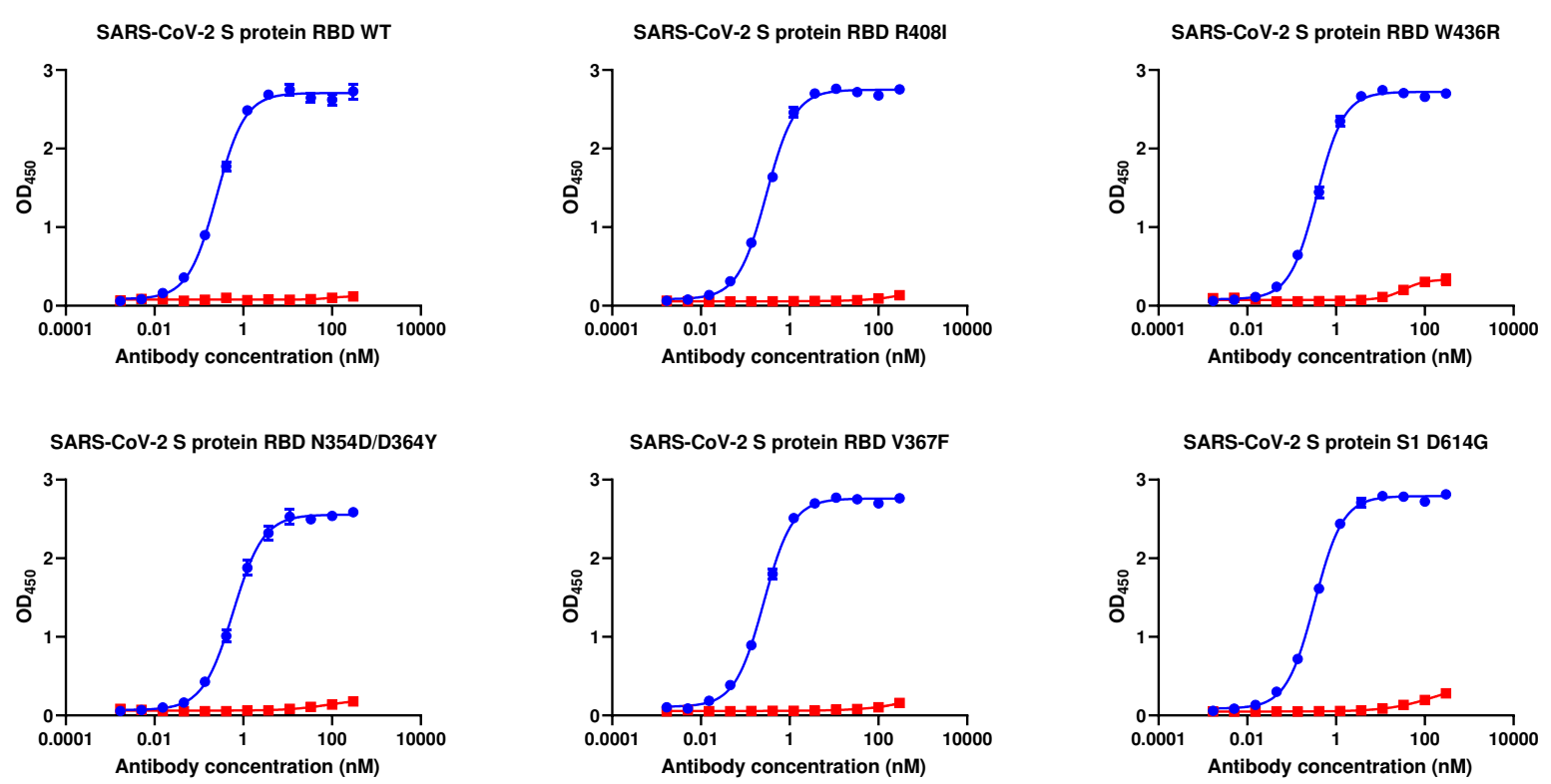

\begin{tabular}{|c|c|c|c|c|c|c|}
\hline & WT & R408I & W436R & N354D/D364Y & V367F & D614G \\
\hline $\mathrm{EC}_{50}(\mathrm{nM})$ & 0.250 & 0.299 & 0.369 & 0.587 & 0.259 & 0.329 \\
\hline
\end{tabular}

376 Fig. S5. The binding of the P4A1-2A (blue) or isotype control (red) antibodies to the

377 SARS-CoV-2 S protein RBD or S1 variants was determined by ELISA. The 384-well

378 plates were coated with $20 \mathrm{nM}$ of the respective SARS-CoV-2 S protein RBD/S1 variants.

379 The binding of P4A1-2A or isotype control antibodies (12 concentrations obtained by 3 -fold 380 serial dilutions of a $300 \mathrm{nM}$ antibody stock solution, in triplicate) was detected by goat $F\left(a b^{\prime}\right) 2$ 381 anti-human IgG $(\mathrm{H}+\mathrm{L})-\mathrm{HRP}$. Table: summary of $\mathrm{EC}_{50}$ s calculated by non-linear regression of 382 the data. 
A
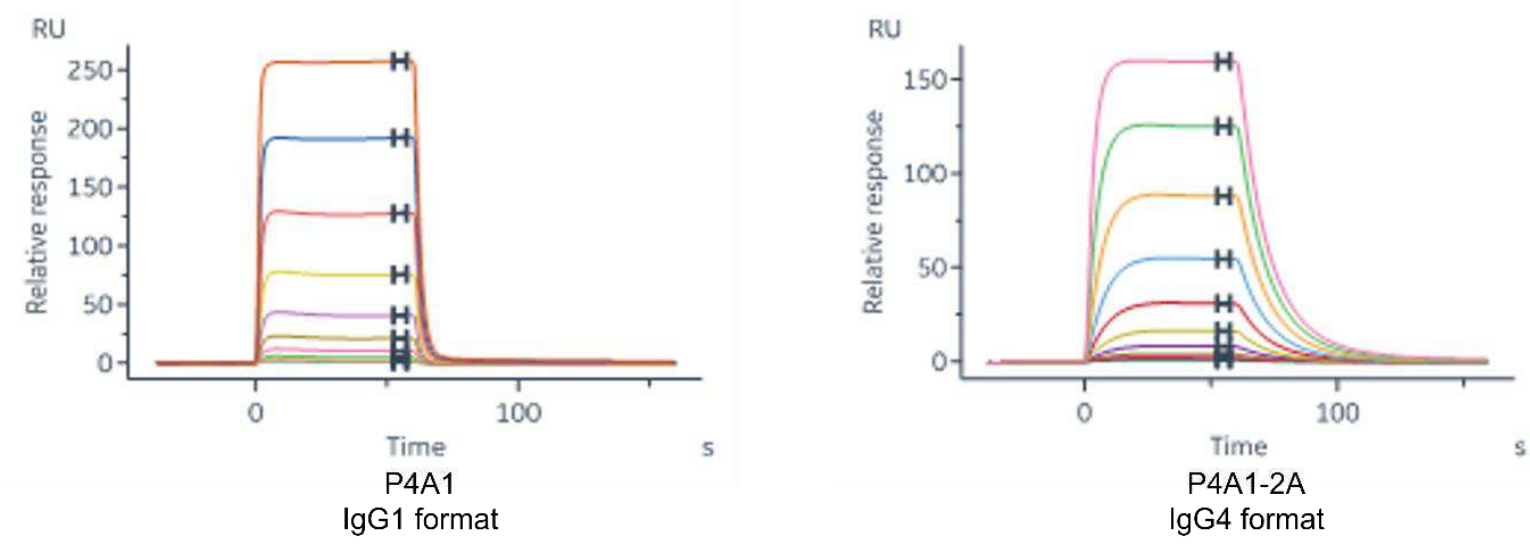

B
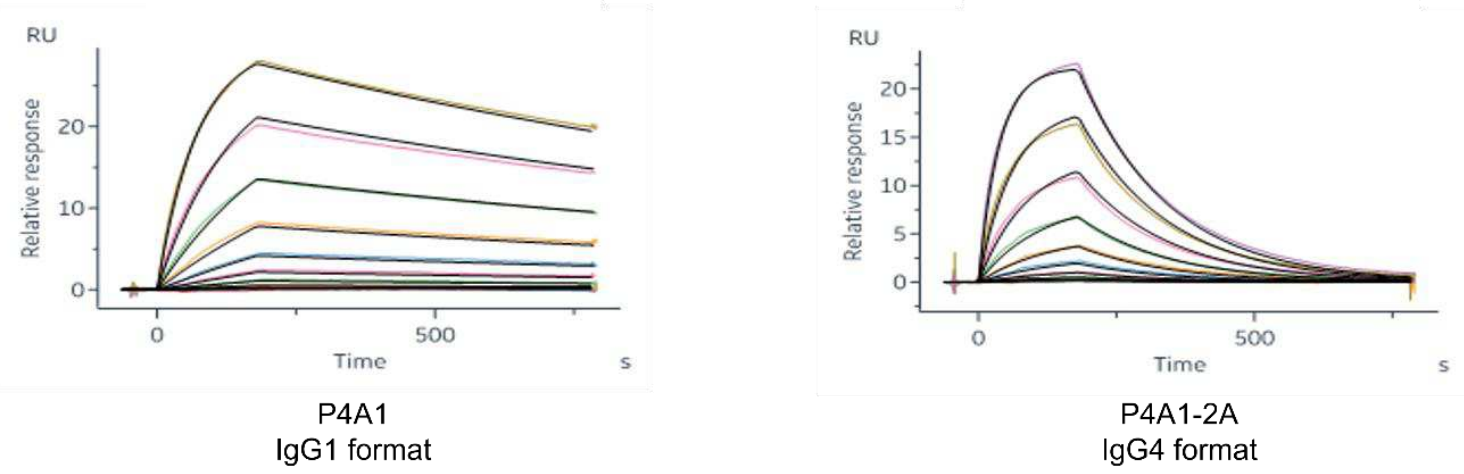

$\mathrm{C}$

Human C1q titration binding to WBP2279.Abs

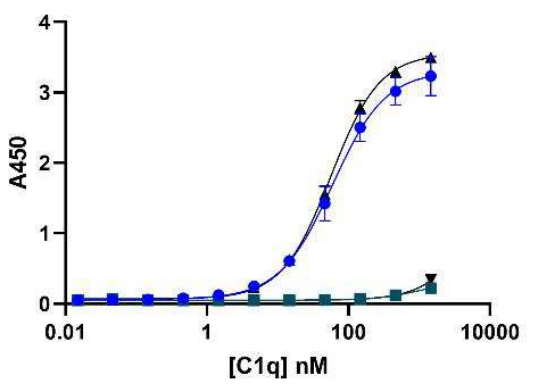

- P4A1, lgG1 format

- P4A1-2A, IgG4 format

$\star$ Human lgG1 isotype control

$\rightarrow$ Human lgG4 isotype control

Fig.S6. Binding of P4A1 (IgG1 form) and P4A1-2A (IgG4 form) to FcRn (A), FcyRI (B)

by SPR using Biacore $8 \mathrm{~K}$ and C1q (C) by ELISA. Assays are detailed in material and methods. 


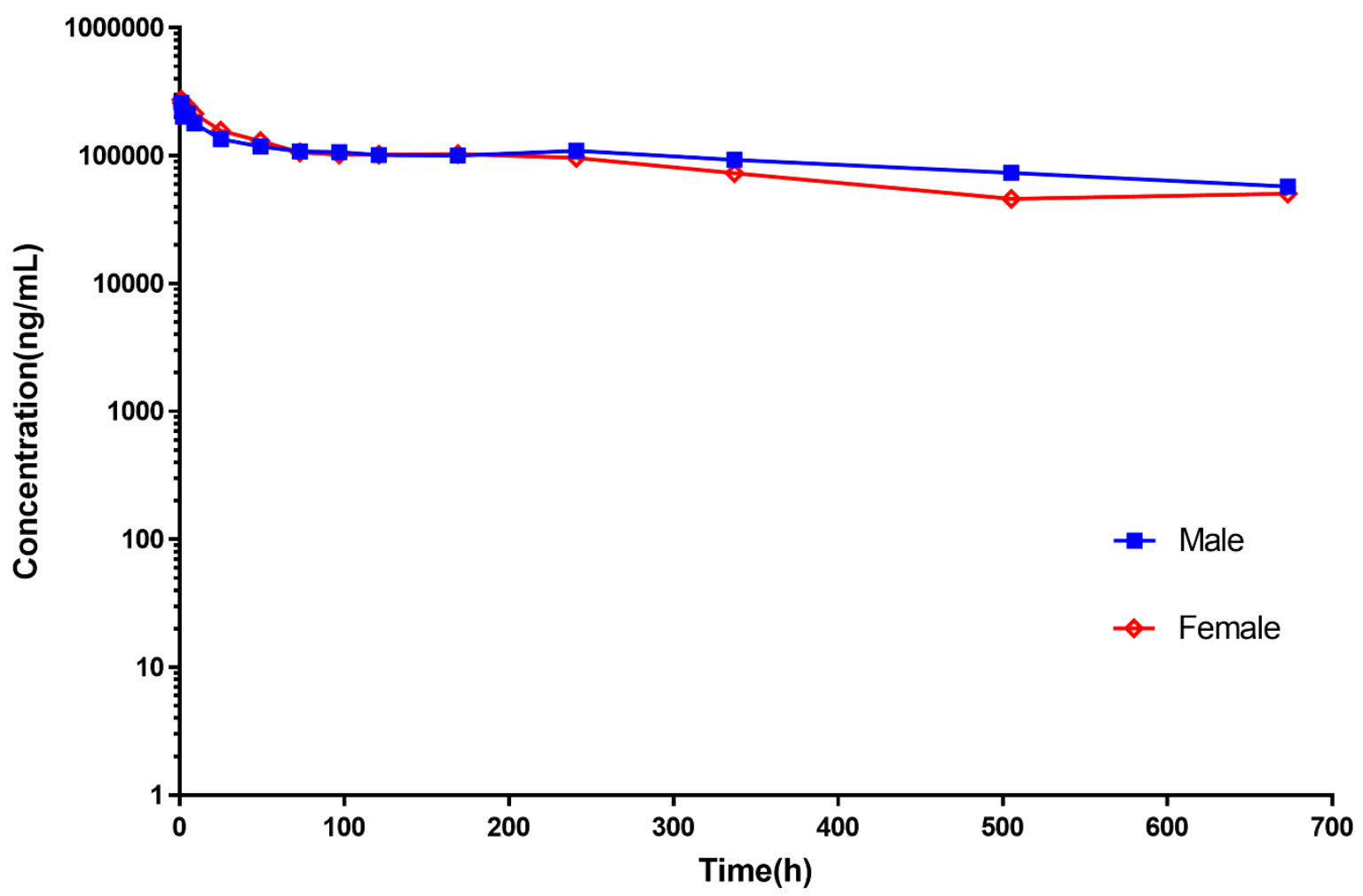

\begin{tabular}{|l|ll|}
\hline PK Parameter & Mean (Male) & Mean (Female) \\
\hline $\mathrm{C}_{\max }(\mathrm{ng} / \mathrm{mL})$ & 259000 & 273000 \\
\hline $\mathrm{C}_{\text {last }}(\mathrm{ng} / \mathrm{mL})$ & 27700 & 13000 \\
\hline $\mathrm{T}_{\max }(\mathrm{h})$ & 1.0833 & 1.0833 \\
\hline $\mathrm{T}_{1 / 2}(\mathrm{~h})$ & 586 & 332 \\
\hline $\mathrm{AUC}_{0 \text {-last }}(\mathrm{ng} . \mathrm{h} / \mathrm{mL})$ & 88200000 & 66700000 \\
\hline
\end{tabular}

Fig.S7. Mean serum P4A1-2A concentration in cynomolgus monkeys. Male and female $\mathrm{mg} / \mathrm{kg}$ P4A1-2A in 60 mins infusion time. Blood samples collected at different timepoints and serum concentrations of P4A1-2A were determined with ELISA. PK parameters derived are listed in the lower panel.

400 
A
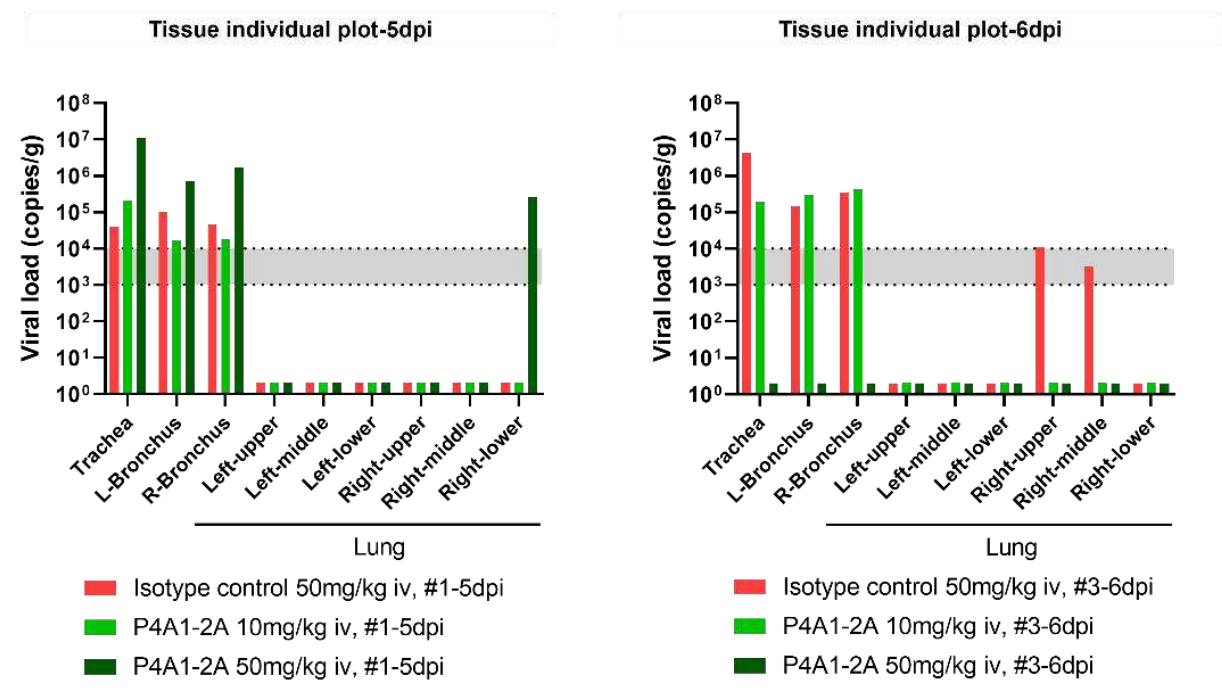

B
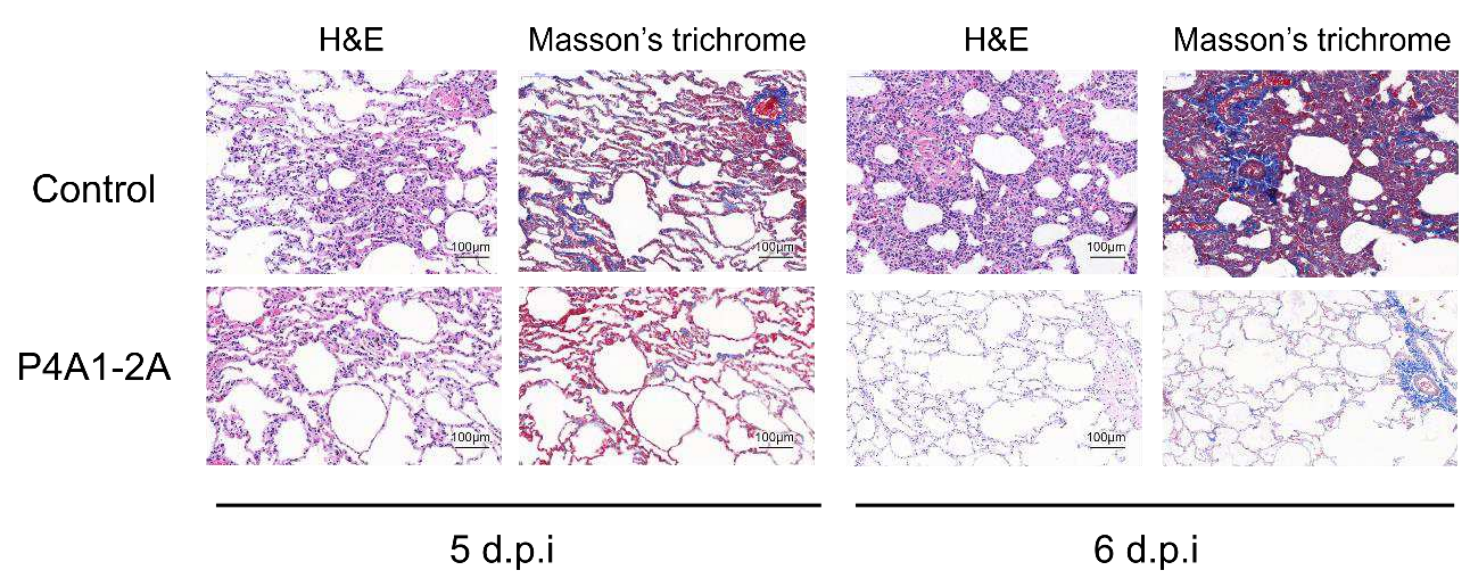

Fig.S8. Viral load and histopathology in lung tissue in the rhesus macaque model of

SARS-CoV-2 infection 5 and 6 d.p.i. (A) Viral load in the respiratory tissues (including trachea, left and right bronchus, and all six lung lobes) collected at necropsy on 5 (left) or 6 408 (right) days post-infection (d.p.i) were tested by RT-qPCR. (B) Representative images of 409 histopathology in lung tissue from isotype control or P4A1-2A $50 \mathrm{mg} / \mathrm{kg}$ treated animals 410 (collected at 5 and 6 d.p.i). 
411 Table S1. Data collection and refinement statistics.

\begin{tabular}{|c|c|}
\hline Wavelength & 0.97915 \\
\hline Resolution range & $36.16-2.108(2.183-2.108)$ \\
\hline Space group & C 2221 \\
\hline Unit cell & 85.958148 .575144 .655909090 \\
\hline Total reflections & 475973 \\
\hline Unique reflections & $48014(4316)$ \\
\hline Multiplicity & $9.6(9.7)$ \\
\hline Completeness (\%) & $89.21(81.11)$ \\
\hline Mean I/sigma(I) & $23.5(2.3)$ \\
\hline Wilson B-factor & 32.62 \\
\hline R-merge & $0.091(0.800)$ \\
\hline R-meas & $0.097(0.844)$ \\
\hline R-pim & $0.031(0.264)$ \\
\hline $\mathrm{CC} 1 / 2$ & $0.996(0.895)$ \\
\hline Reflections used in refinement & 47909 (4316) \\
\hline Reflections used for R-free & $1991(185)$ \\
\hline R-work & $0.1858(0.2309)$ \\
\hline R-free & $0.2284(0.2616)$ \\
\hline Number of non-hydrogen atoms & 5466 \\
\hline macromolecules & 4861 \\
\hline ligands & 14 \\
\hline solvent & 591 \\
\hline Protein residues & 793 \\
\hline RMS(bonds) & 0.007 \\
\hline RMS(angles) & 0.95 \\
\hline Ramachandran favored (\%) & 97.1 \\
\hline Ramachandran allowed (\%) & 2.9 \\
\hline Ramachandran outliers (\%) & 0 \\
\hline Rotamer outliers (\%) & 0.55 \\
\hline Clashscore & 4.8 \\
\hline Average B-factor & 39.13 \\
\hline macromolecules & 37.67 \\
\hline ligands & 82.89 \\
\hline solvent & 50.16 \\
\hline Number of TLS groups & 1 \\
\hline
\end{tabular}

412 Statistics for the highest-resolution shell are shown in parentheses. 
Table S2. Residues contributed to interaction between P4A1/SARS-CoV-2-RBD.

\begin{tabular}{|c|c|c|c|c|}
\hline SARS-CoV-2 RBD & \multicolumn{3}{|c|}{ 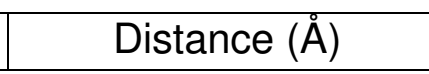 } & P4A1 Antibody \\
\hline \multicolumn{5}{|c|}{ Hydrogen Bonds } \\
\hline SER 477 [N ] & \multicolumn{3}{|c|}{3.1} & VH:GLY 26 [O] \\
\hline LYS 458 [NZ] & \multicolumn{3}{|c|}{2.7} & VH:SER 30 [O] \\
\hline TYR 473 [OH] & \multicolumn{3}{|c|}{2.8} & VH:SER 31 [O] \\
\hline TYR 449[OH] & \multicolumn{3}{|c|}{4.0} & VH: SER 31[OG] \\
\hline LYS 458 [NZ] & \multicolumn{3}{|c|}{3.1} & VH:SER 53 [O] \\
\hline TYR $421[\mathrm{OH}]$ & \multicolumn{3}{|c|}{3.4} & VH:SER 53 [OG] \\
\hline LYS 417 [NZ] & \multicolumn{3}{|c|}{2.7} & VH: GLN 100 [OE1] \\
\hline TYR $453[\mathrm{OH}]$ & \multicolumn{3}{|c|}{2.4} & VH: GLU 101 [OE1] \\
\hline ASP 420 [OD2] & \multicolumn{3}{|c|}{2.5} & VH:SER 56 [OG] \\
\hline TYR $421[\mathrm{OH}]$ & \multicolumn{3}{|c|}{3.6} & VH:SER 53 [N] \\
\hline TYR $421[\mathrm{OH}]$ & \multicolumn{3}{|c|}{2.9} & VH:GLY 54 [N] \\
\hline LEU 455 [O] & \multicolumn{3}{|c|}{2.8} & VH:TYR $33[\mathrm{OH}]$ \\
\hline ARG 457 [O] & \multicolumn{3}{|c|}{2.6} & VH:SER 53 [OG] \\
\hline ALA $475[\mathrm{O}]$ & \multicolumn{3}{|c|}{3.2} & VH:ILE 28 [N] \\
\hline ALA $475[0]$ & \multicolumn{3}{|c|}{2.9} & VH:ASN 32 [ND2] \\
\hline ASN 487 [OD1] & \multicolumn{3}{|c|}{2.9} & VH:ARG 97 [NH1] \\
\hline TYR 489 [OH] & \multicolumn{3}{|c|}{3.4} & VH:ARG 97 [NH2] \\
\hline GLY 502 [N] & \multicolumn{3}{|c|}{3.0} & VL:GLY 28 [O] \\
\hline ARG 403 [NH1] & \multicolumn{3}{|c|}{2.9} & VL:ASN 92 [O] \\
\hline ARG 403 [NH2] & \multicolumn{3}{|c|}{3.1} & VL:ASN $92[\mathrm{O}]$ \\
\hline TYR $505[\mathrm{OH}]$ & \multicolumn{3}{|c|}{2.7} & VL:SER 93 [OG] \\
\hline ASN 501 [OD1] & \multicolumn{3}{|c|}{3.2} & VL:SER 30 [N] \\
\hline GLY 496 [O] & \multicolumn{3}{|c|}{3.0} & VL:SER 30 [OG] \\
\hline ASN 501 [OD1] & \multicolumn{3}{|c|}{3.0} & VL:SER 30 [OG] \\
\hline GLN 498 [OE1] & \multicolumn{3}{|c|}{3.4} & VL:SER 67 [OG] \\
\hline TYR 505 [OH] & \multicolumn{3}{|c|}{3.6} & VL:SER 93 [N] \\
\hline \multicolumn{5}{|c|}{ Salt Bridge } \\
\hline LYS 417 [NZ] & & 3.1 & & VH: GLU 101 [OE2] \\
\hline & vent $\mathrm{r}$ & drogen B & nd $\mathrm{Br}$ & \\
\hline GLY 476 [O] & 3.0 & W740 & 3.0 & VH: SER 31 [OG] \\
\hline TYR $505[\mathrm{OH}]$ & 2.8 & W388 & 2.8 & VL: TRP 32[O] \\
\hline TYR $505[\mathrm{OH}]$ & 2.8 & W388 & 3.1 & VL: ALA $91[\mathrm{~N}]$ \\
\hline TYR $505[\mathrm{OH}]$ & 2.8 & W388 & 2.9 & VL: ASN $92[\mathrm{~N}]$ \\
\hline
\end{tabular}

415 


\section{Reference.}

418 1. A. Gérard et al., High-throughput single-cell activity-based screening and sequencing 419 of antibodies using droplet microfluidics. Nature Biotechnology 38, 715-721 (2020).

420 2. P. Zhou et al., A pneumonia outbreak associated with a new coronavirus of probable $421 \quad$ bat origin. Nature 579, 270-273 (2020).

422 3. W. Minor, M. Cymborowski, Z. Otwinowski, M. Chruszcz, HKL-3000: the integration 423 of data reduction and structure solution--from diffraction images to an initial model in 424 minutes. Acta Crystallogr D Biol Crystallogr 62, 859-866 (2006).

425 4. A. J. McCoy et al., Phaser crystallographic software. J Appl Crystallogr 40, 658-674 $426 \quad$ (2007).

427 5. J. Lan et al., Structure of the SARS-CoV-2 spike receptor-binding domain bound to 428 the ACE2 receptor. Nature 581, 215-220 (2020).

429 6. P. Emsley, B. Lohkamp, W. G. Scott, K. Cowtan, Features and development of Coot. $430 \quad$ Acta Crystallogr D Biol Crystallogr 66, 486-501 (2010).

431 7. P. D. Adams et al., PHENIX: a comprehensive Python-based system for 432 macromolecular structure solution. Acta Crystallogr D Biol Crystallogr 66, 213-221 433 (2010).

434 8. Schrodinger, LLC. (2015).

435 9. C. Shan et al., Infection with novel coronavirus (SARS-CoV-2) causes pneumonia in 436 Rhesus macaques. Cell Res 30, 670-677 (2020). 


\section{Figures}

A

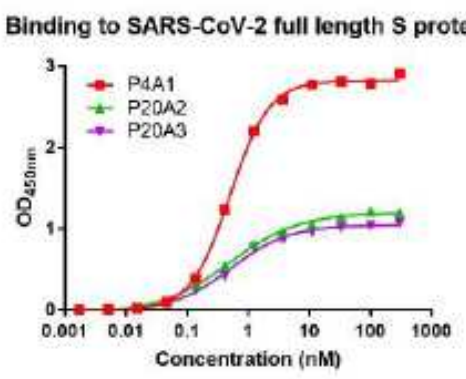

Binding to SARS-CoV-2 S1-mFc protein

Binding to SARS-CoV-2 S2-his protein
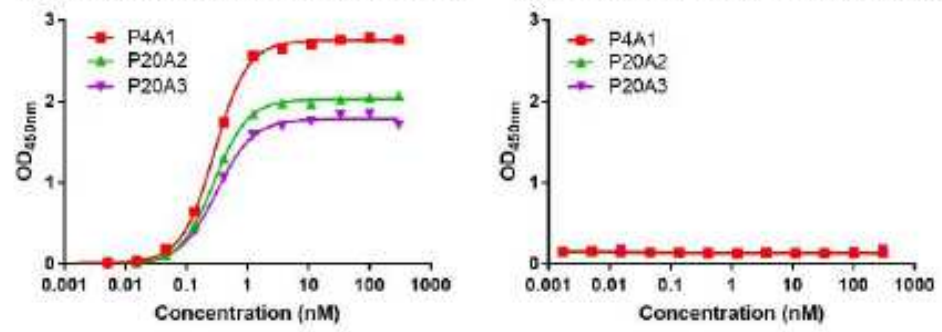

Inhibition of S1 binding to Vero-E6
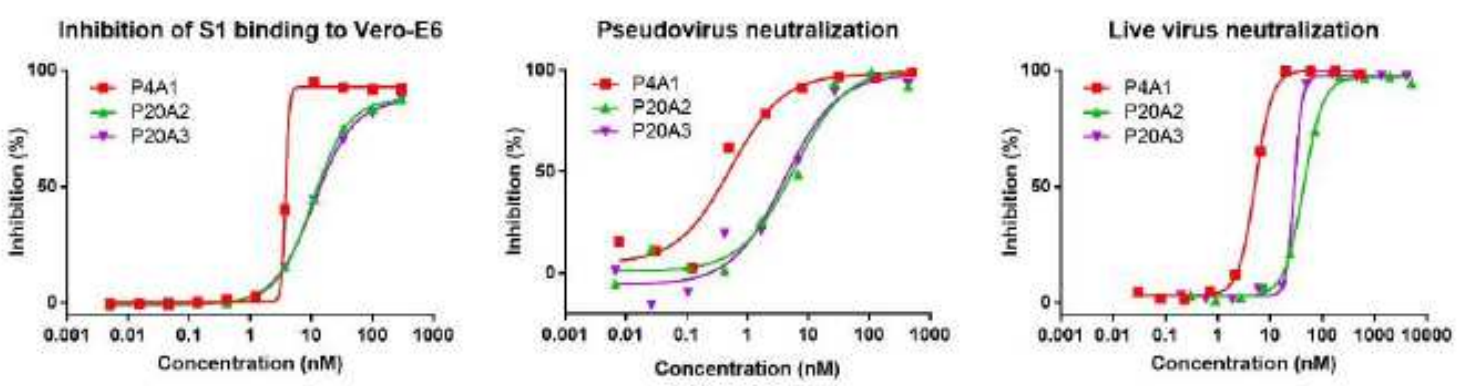

B

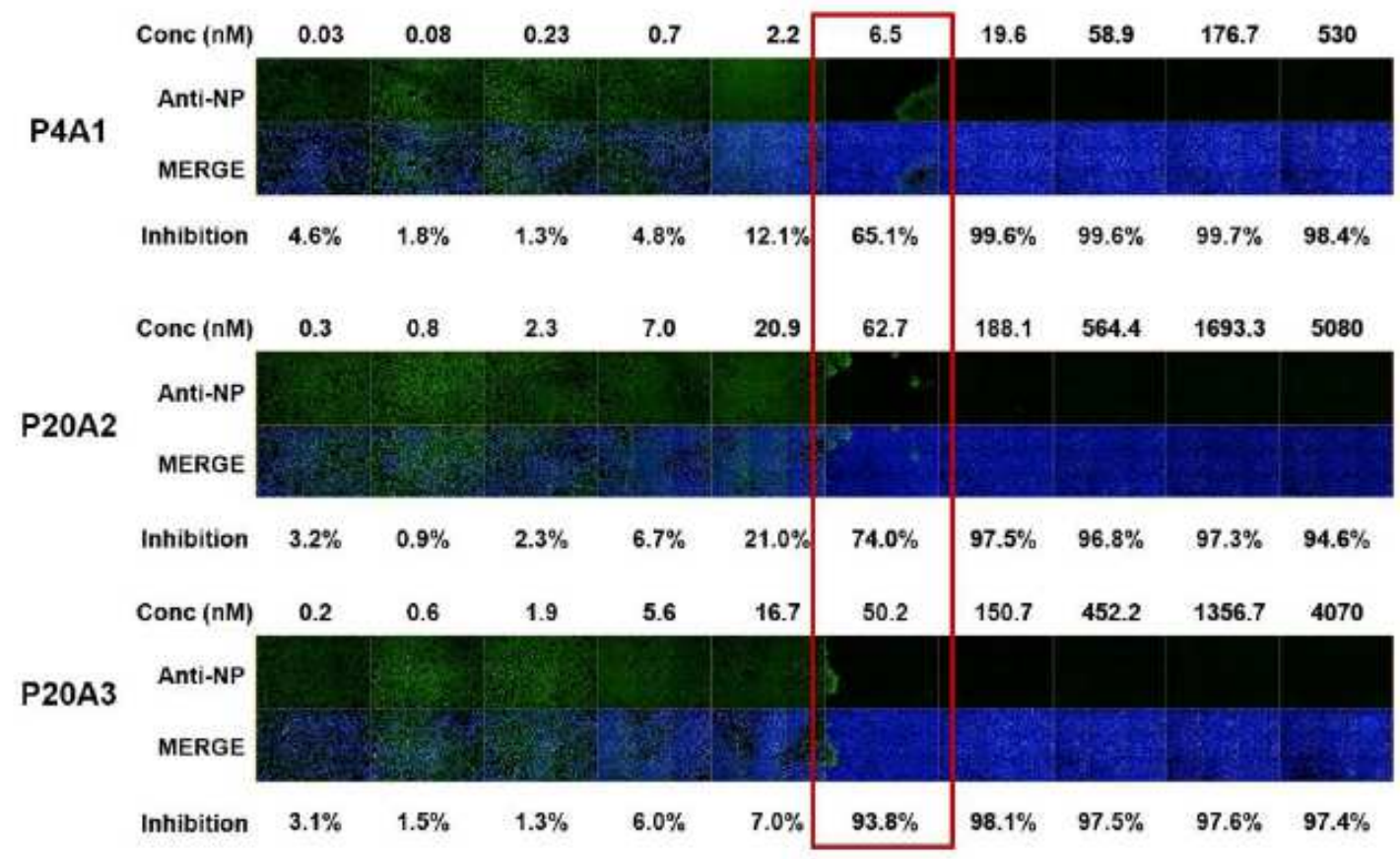

Figure 1

Characterization of neutralizing antibodies from convalescent patients. (A) Characterization of SARSCoV-2 S protein specific antibodies. (upper panels) Binding of antibodies to the full-length S protein, S1 protein and S2 protein were evaluated by ELISA. (lower left panel) Blockage of the binding of SARS-CoV-2 
spike S1 protein to Vero E6 cell line by antibodies evaluated by flow cytometry. (Lower middle panel) Pseudovirus neutralization assay in Huh-7 cell line. (lower right panel and B) SARS-CoV-2 live virus neutralization assay. (B) Images of Vero E6 cells infected SARS-CoV-2 treated with antibodies of different concentrations.
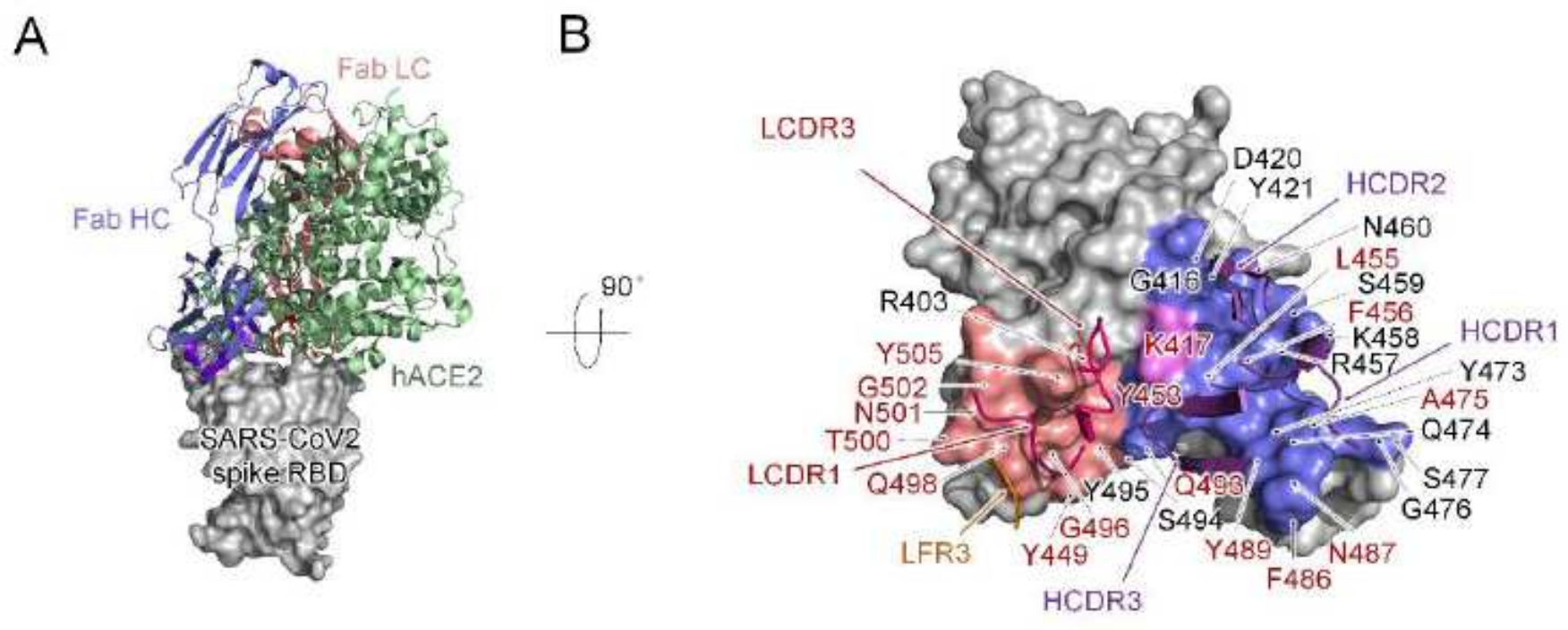

C
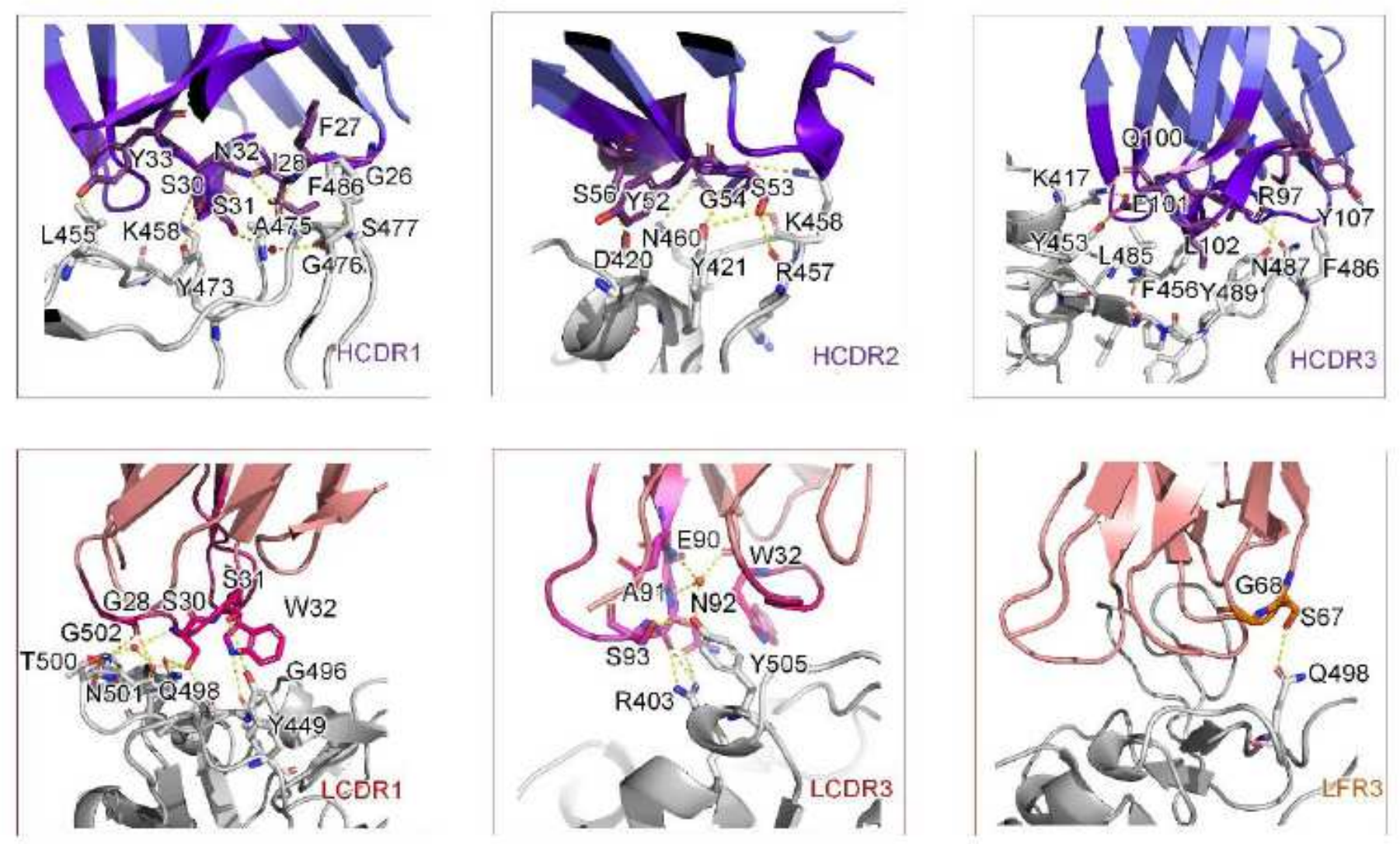

Figure 2

Structural analysis of P4A1 Fab and SARS-CoV-2 RBD complex. (A) The overall P4A1-Fab-RBD complex structure superimposed with the hACE2-RBD complex. The P4A1 heavy chain (colored slate blue), light 
chain (colored salmon red) and hACE (colored pale green) are displayed in cartoon representation. The SARS-CoV-2 RBD is colored in gray and displayed in surface representation. (B) The epitope of P4A1 shown in surface representation. The CDR loops of heavy chain (HCDR) and light chain (LCDR) are colored in purple and magenta, respectively. The epitopes from the heavy chain and light chain are colored in slate blue and salmon red, respectively. The only residue K417, which contacts with both heavy chain and light chain is colored in pink. The light chain frame region 3 (LFR3) is colored in orange. The identical residues on RBD shared in P4A1 and hACE2 binding are labelled in red. The residues are numbered according to SARS-CoV-2 RBD. (C) The detailed interactions between SARS-CoV-2 RBD with HCDR, LCDR and LFR3. The residues are shown in sticks with identical colors to (B).

A
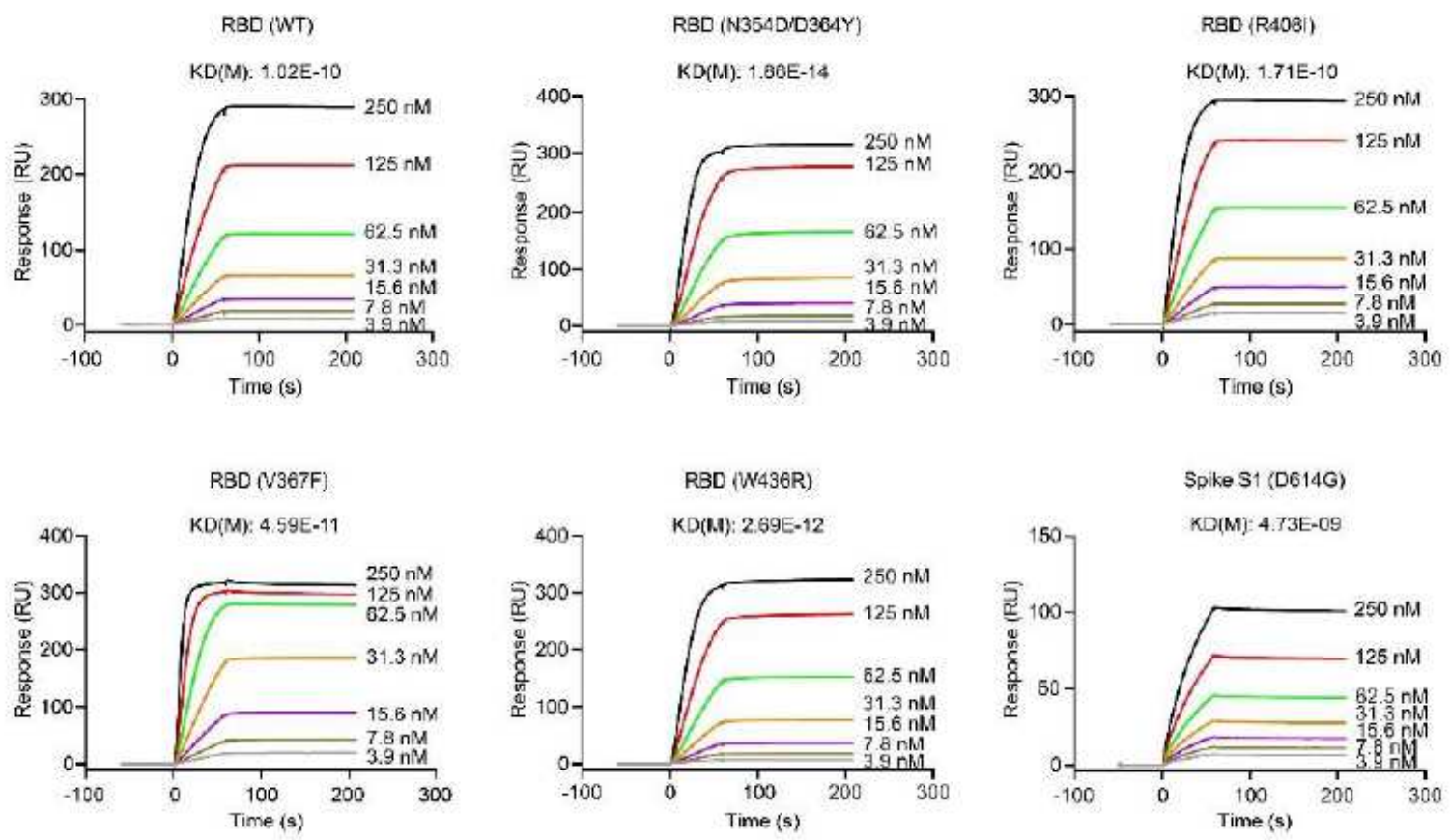

B
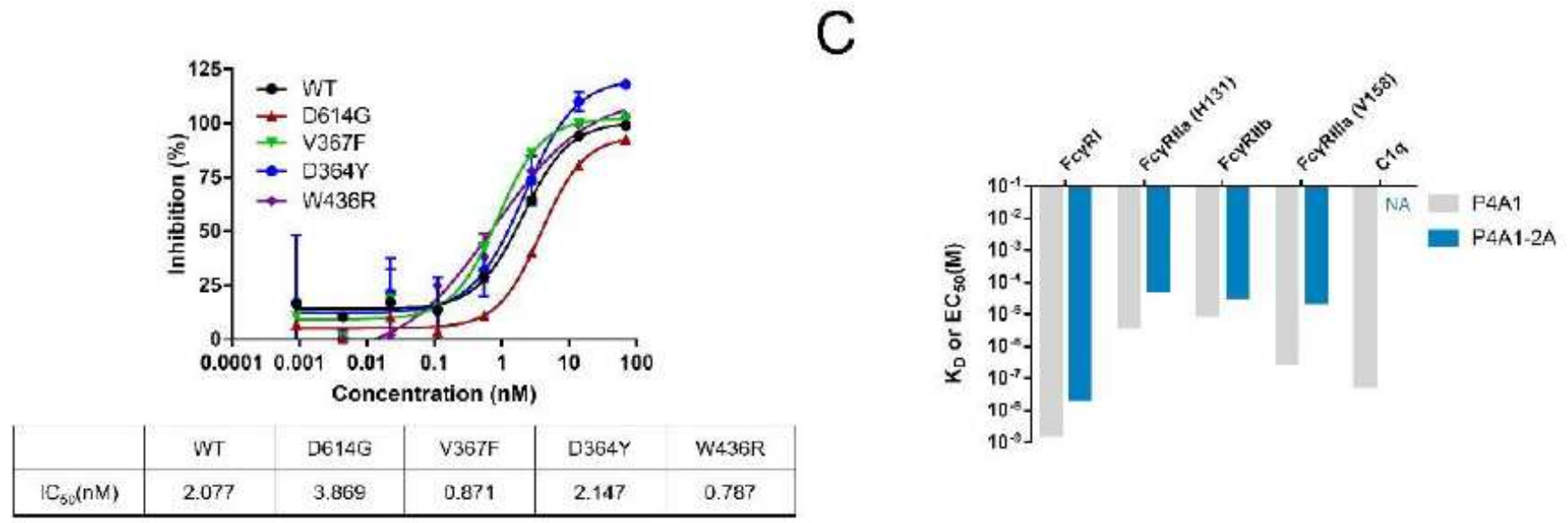

Figure 3 
The activities of IgG4 antibody P4A1-2A to different SARS-CoV-2 S protein mutants, FcyRs and C1q. (A) Binding of antibody P4A1 to SARS-CoV-2 S protein N354D/D364Y, R408I, W436R, V367F or D614G mutants determined by SPR. (B) Pseudo virus neutralization assay in hACE2 overexpressing HEK293 cells. (C) The binding affinity of P4A1 and P4A1-2A for different human FcyRs and complement C1q.

A

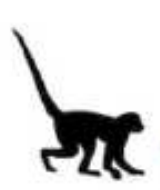

$\begin{array}{llllllllllll}\text { Day } & -3 & -2 & -1 & 0 & 1 & 2 & 3 & 4 & 5 & 6 & 7 \\ 0 & & & & & & & & & & & \end{array}$

Virus infection $\because$ Antibody injection Daily monitor

Euthanized and necropsy

o X-ray examination

B

Viral Load in Oropharyngeal Swabs
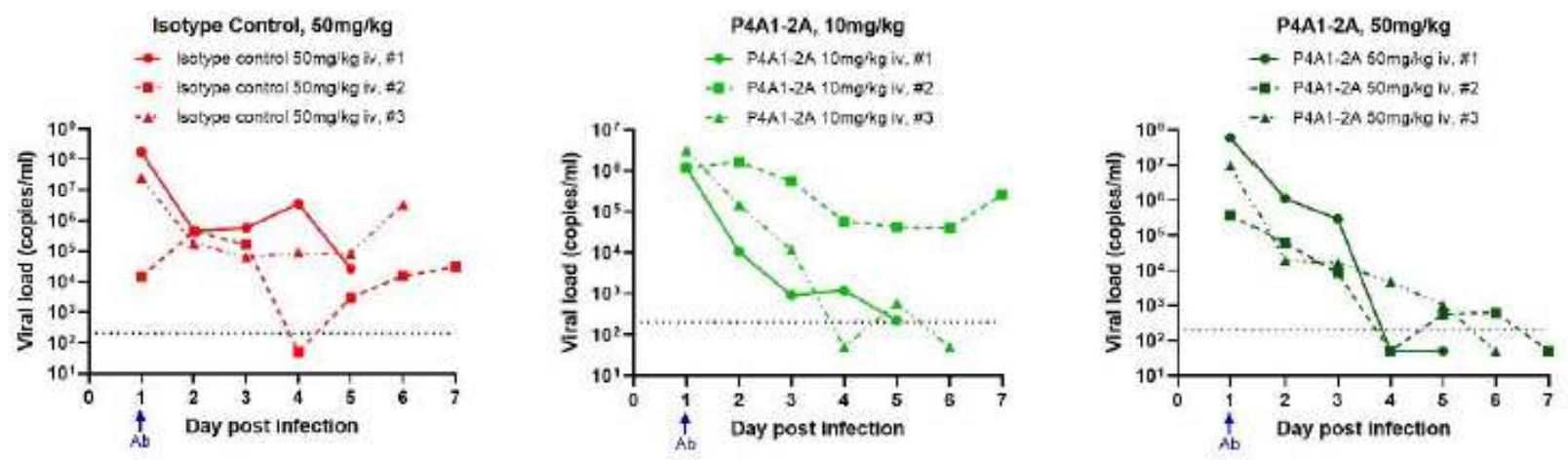

C

Tissue individual plot-7dpi

D
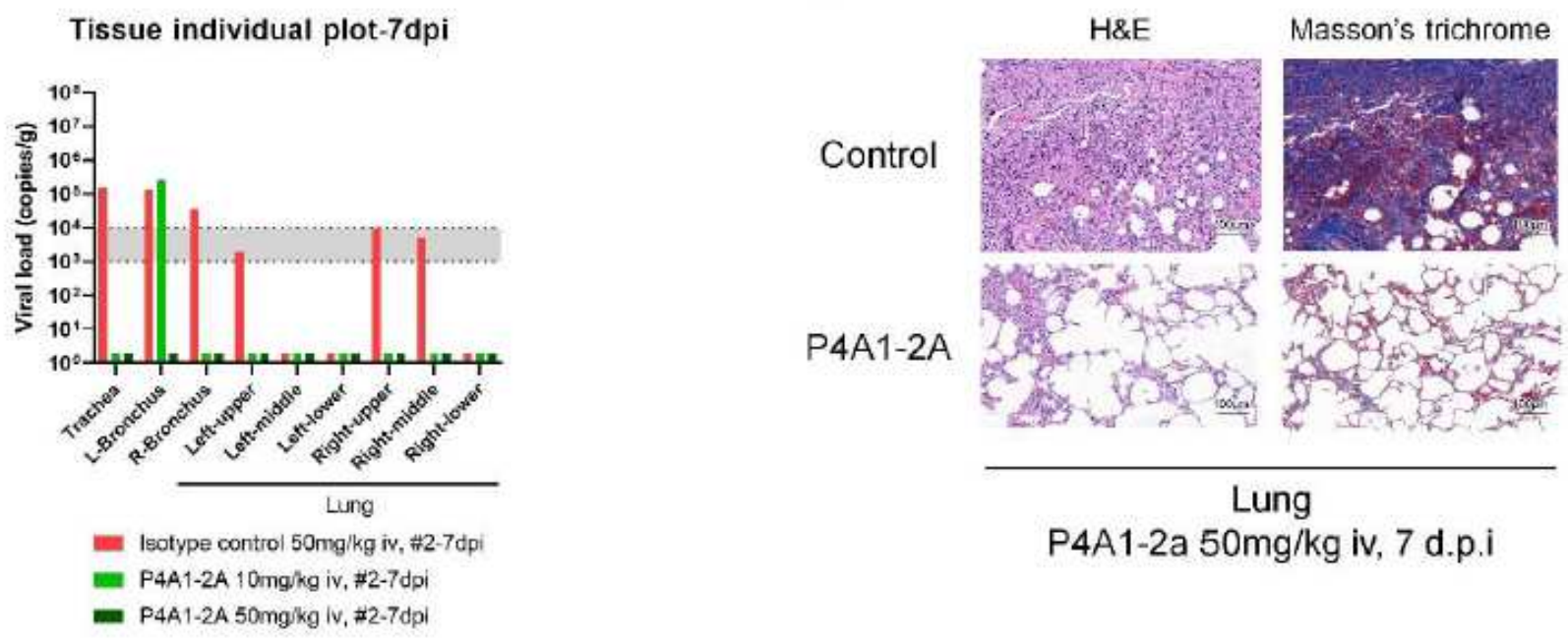

Figure 4

Therapeutic efficacy of in the rhesus macaque model of SARS-CoV-2 infection. (A) Experimental design for therapeutic testing of P4A1-2A in the rhesus macaque. (B) Viral load in oropharyngeal swabs tested 
by RT-qPCR were monitored for 7 days. (C) Viral load in the respiratory tissues (including trachea, left and right bronchus, and all six lung lobes) collected at necropsy on 7 days post-infection (d.p.i) were tested by RT-qPCR. (D) Representative images of histopathology in lung tissue from isotype control or P4A1-2A 50 $\mathrm{mg} / \mathrm{kg}$ treated animals (collected at 7 d.p.i).

\section{Supplementary Files}

This is a list of supplementary files associated with this preprint. Click to download.

- NCOMMS2041615T.pdf

- NCOMMS2041615TvalreportfullP1.pdf 